TRANSACTIONS OF THE

AMERICAN MATHEMATICAL SOCIETY

Volume 301, Number 2, June 1987

\title{
INDUCED GROUP ACTIONS, REPRESENTATIONS AND FIBERED SKEW PRODUCT EXTENSIONS
}

\author{
R. C. FABEC
}

\begin{abstract}
Let $G$ be a locally compact group acting ergodically on $Y$. We introduce the notion of an action of this group action and study the notions of induced group actions, ergodicity, and fibered product extensions in this context. We also characterize fibered skew product actions built over a cocycle.
\end{abstract}

This paper is essentially a continuation of our earlier papers [2, 3]. There we were concerned with the problem of studying the interrelationships among the geometric structure of an extension of an ergodic group action, the Hilbert bundle representation it induces, and a cocycle on the group action having Mackey dense range in a locally compact group. In [3] we showed that an extension is a skew product extension built over a cocycle $\phi$ having Mackey dense range if and only if the restriction of the natural bundle representation to the Mackey 'kernel' of $\phi$ is trivial. We also studied cocycle representations which have trivial restrictions to Mackey 'kernels'. In this paper we remove the stricture that the cocycle have dense range. Doing this leads one to consider the notion of an action of an ergodic group action and the virtual group notion of a homomorphism between ergodic group actions. The former is equivalent with the notion of an extension of the action. In [25], Zimmer showed that an action of a subgroup may be used to induce an action of the group. We show this is a natural special case for a similar method of inducing an action from an action of a group action. If one uses the equivalent notion of an extension, the induced action is the extension. Moreover, in the case of locally compact groups, homomorphisms may be used to 'pull back' actions and representations. The 'pull backs' of actions induce fibered skew product extensions. Before proceeding with our discussion we introduce Mackey's notion of a virtual subgroup. This notion, though not necessary for the presentation of this paper, was central in the development of its ideas.

Suppose $G$ is a locally compact, separable group and $X$ is a standard Borel $G$ space with an ergodic, quasi-invariant measure $\mu$. Then either $X$ has a conull orbit or every orbit in $X$ is null. In the first case, $X$ is essentially a coset space $H \backslash G$, where $H$ is the stabilizer of some point in the conull orbit, and the action is given by right translation. The subgroup $H$ is determined up to conjugacy and is "identified" with the measure groupoid ( $H \backslash G \times G, \mu \times m$ ), where $m$ is a Haar measure on $G$ and multiplication is defined by $(x, g) \cdot\left(x \cdot g, g^{\prime}\right)=\left(x, g g^{\prime}\right)$. The "identification"

Received by the editors May 13, 1985 and, in revised form, May 14, 1986.

1980 Mathematics Subject Classification (1985 Revision). Primary 24D80, 28 D15.

(C)1987 American Mathematical Society $0002-9947 / 87 \$ 1.00+\$ .25$ per page 
is given by the multiplication preserving map $I$ defined on $H \backslash G \times G$ by $(x, g) \rightarrow$ $\gamma(x) g \gamma(x \cdot g)^{-1}$, where $\gamma$ is a Borel map on $H \backslash G$ into $G$ satisfying $H \gamma(x)=x$. If, in addition, $\gamma(H)=e$, the map $i$ from $H \backslash G \times G$ into $G$ sending $(x, g)$ to $g$ factors through $I$.

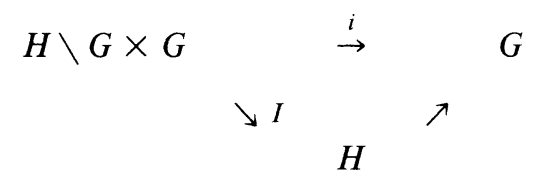

Hence thinking of $I$ as an "identification" corresponds the map $i$ to the inclusion map of $H$ into $G$. Thus, in this case, the "virtual subgroup" of $G$ defined by the ergodic action is a conjugacy class of closed subgroups, and one sees the virtual subgroup actually is a subgroup.

In the second case one cannot find a conjugacy class of closed subgroups, but one can still define the measure groupoid $(X \times G, \mu \times m)$ and the map $i$ sending $(x, g)$ to $g$. This measure groupoid is said to define a virtual subgroup of $G$, and the function $i$ is called the inclusion. Hence the ergodic actions of $G$ determine the virtual subgroups of $G$. Two ergodic actions define the same virtual subgroup of $G$ if the actions are essentially isomorphic.

Suppose $(X \times G, \mu \times m)$ and $(Y \times G, \nu \times m)$ are virtual subgroups of $G$. Then $X$ and $Y$ are ergodic $G$ spaces. $X \times G$ is said to be a subvirtual subgroup of the virtual subgroup $Y \times G$ if $X$ is an extension of the $G$ space $Y$. Moreover, if $p$ is an extension, that is, an equivariant Borel mapping from $X$ into $Y$ satisfying $p_{*} \mu \equiv$ $\mu \circ p^{-1} \sim \nu$, then the map $(x, g) \rightarrow(p x, g)$ is called the inclusion of $X \times G$ in $Y \times G$. Thus ergodic extensions of ergodic group actions correspond to inclusions between virtual subgroups.

There are several notions from the theory of locally compact groups which can be extended to the virtual subgroup setting that will play significant roles in this paper. These include the notions of group actions, homomorphisms and their restrictions and ranges, representations, and induced representations. We define these in our first section. Moreover, in $\$ 1$ we develop the notion of an action of a group action and the related concepts of ergodicity and induction. We determine the relationship between this notion and that of an extension. We then study the compositions of actions and representations with homomorphisms. These are called pull backs. The pull back actions induce skew fiber product extensions. In $\S 2$ we obtain characterizations for actions and representations which are equivalent to pull backs. These allow us to obtain structure theorems for skew product fibered extensions and representations which induce trivial extensions over a homomorphism. The appendix contains some technical results used throughout this paper as well as a description of the construction of the Mackey range of a homomorphism.

The theory of virtual subgroups was begun by Mackey to treat the nonregular case in his analysis of the dual of $G$ in terms of the action of $G$ on the dual of $N$, where $N$ is a normal subgroup. He sketched many of the ideas for this theory in [9]. These have been developed in further detail by Ramsay [13-16]. In general we follow the terminology of [13]. 
1. Actions, homomorphisms, and representations. Let $G$ be a second countable, locally compact group. Suppose $\boldsymbol{\nu}$ is a $\boldsymbol{\sigma}$-finite measure on a standard Borel space $Y$. Then $Y$ is a strict $G$ space provided there exists a Borel mapping $(y, g) \rightarrow y \cdot g$ satisfying

(1.1) $y \cdot e=y$ for all $y$,

(1.2) $y \cdot g_{1} \cdot g_{2}=y \cdot g_{1} g_{2}$ for all $y, g_{1}$, and $g_{2}$, and

(1.3) $y \mapsto y \cdot g$ preserves the measure class of $\nu$ for each $g$.

Two $G$ spaces $\left(Y_{1}, \nu_{1}\right)$ and $\left(Y_{2}, \nu_{2}\right)$ are isomorphic provided there exists a Borel isomorphism $\Phi: Y_{1} \rightarrow Y_{2}$ such that $\Phi(y \cdot g)=\Phi(y) \cdot g \nu_{1}$ a.e. $y$ for each $g$ and $\Phi_{*} \nu_{1} \sim \nu_{2}$.

One can give an alternate definition for a $G$ space if one first introduces the notion of a point transformation group. Suppose $S$ is a standard Borel space with a finite measure $m$. Let $\mathscr{I}(S, m)$ be the set of all Borel isomorphisms of $S$ which preserve the measure class of $m$, identified when they agree pointwise a.e. $m$. Give $\mathscr{I}(S, m)$ the Borel structure defined by convergence in measure. Then $\mathscr{I}(S, m)$ is a standard Borel group. In fact, the map $\phi \rightarrow L_{\phi}$ where

$$
\left(L_{\phi} f\right)(s)=\frac{d m \circ \phi^{-1}}{d m}(s)^{1 / 2} f\left(\phi^{-1} s\right)
$$

defines a Borel isomorphism of $\mathscr{I}(S, m)$ onto the strongly closed subgroup of the unitary group of $L^{2}(S)$ consisting of the unitary operators $U$ satisfying $U 1 \geqslant 0$ and $U L^{\infty}(S) U^{-1}=L^{\infty}(S)$, where $L^{\infty}(S)$ is the von Neumann algebra consisting of all bounded multiplication operators. Thus $\mathscr{I}(S, m)$ is a Polish group.

Now by an action of $G$ on $(Y, \nu)$ we shall mean a Borel homomorphism $a$ from $G$ into $\mathscr{I}(Y, \nu)$. Mackey in [6] showed that if one has such a homomorphism, then there exists a strict $G$ action on $(Y, \nu)$ such that $y \cdot g=a\left(g^{-1}\right) y$ a.e. $y$ for each $g$. In particular, any action $a$ determines an isomorphism class of strict $G$ actions.

We shall follow the second approach to define an action of a group action. To do this we shall need the notion of a cocycle.

Suppose $(Y, \nu)$ is a strict $G$ space and $H$ is a complete separable metric group. A cocycle on the $G$ space $Y$ with values in $H$ is a Borel mapping $\phi$ from $Y \times G$ into $H$ satisfying

$$
\phi\left(y, g_{1} g_{2}\right)=\phi\left(y, g_{1}\right) \phi\left(y \cdot g_{1}, g_{2}\right)
$$

for all $y, g_{1}$, and $g_{2}$.

Identity (1.5) states that $\phi$ is multiplicative on the measure groupoid $Y \times G$. Two cocycles $\phi_{1}$ and $\phi_{2}$ on $Y \times G$ with values in $H$ are cohomologous provided there is a Borel map $A: Y \rightarrow H$ satisfying $A(y) \phi_{1}(y, g)=\phi_{2}(y, g) A(y \cdot g)$ a.e. $(y, g)$.

Suppose $(Y, \nu)$ is a strict $G$ space and $(S, m)$ is a standard Borel space with a finite measure $m$. Then a $Y \times G$ action $a$ on $S$ is defined to be a cocycle $a$ on the $G$ space $Y$ with values in $\mathscr{I}(S, m)$. Two actions $a_{1}$ and $a_{2}$ of the $G$ space $(Y, \nu)$ on $(S, m)$ are said to be isomorphic provided the cocycles $a_{1}$ and $a_{2}$ are cohomologous. 
If the action of $G$ on $Y$ is ergodic, an action of the $G$ space $Y$ is an action of the virtual subgroup $Y \times G$.

We recall if $(X, \mu)$ and $(Y, \nu)$ are strict $G$ spaces, then a Borel map $p: X \rightarrow Y$ is an extension if $p(x \cdot g)=p(x) \cdot g$ for all $(x, g)$ and $p_{*} \mu \sim \nu$. Two extensions $p$ : $(X, \mu) \rightarrow(Y, \nu)$ and $p^{\prime}:\left(X^{\prime}, \mu^{\prime}\right) \rightarrow(Y, \nu)$ are isomorphic over $Y$ if there exists a $G$ space isomorphism $\Phi$ from $X$ to $X^{\prime}$ such that $p^{\prime} \circ \Phi(x)=p(x)$ a.e. $x$.

Zimmer in [26] showed that one can induce an action of a group from an action of a closed subgroup. The same is true for a virtual subgroup. In order to facilitate the construction, we use the notion of the universal extension space over $Y$. This is defined to be the pair $\left(Y \times U^{G}, p_{Y}\right)$ where $U^{G}$ is a universal $G$ space in the sense of Mackey and $p_{Y}$ is the coordinate projection onto $Y$. There is a pointwise action of $G$ on $Y \times U^{G}$; namely $(y, f) \cdot g=(y \cdot g, f \cdot g)$. For $U^{G}$ we take $U^{G}=\left\{f \in L^{\infty}(G)\right.$ : $f(x) \in[0,1]$ a.e. $x\}$ with topology defined by the pseudometrics $\rho_{K}, K$ compact, where $\rho_{K}\left(f_{1}, f_{2}\right)=\int_{K}\left|f_{1}(x)-f_{2}(x)\right|^{2} d x$. The action on $U^{G}$ is defined by $(f \cdot g)(x)$ $=f(g x)$. Then $U^{G}$ is a Polish space and the action is continuous. $Y \times U^{G}$ is a universal extension of $Y$ in the following sense.

Proposition 1.1. Suppose $X$ is a strict $G$ space and $p$ is an equivariant Borel map from $X$ into $Y$. Then there exists a one-to-one Borel map $\Phi$ from $X$ into $Y \times U^{G}$ such that

(a) $\Phi(x \cdot g)=\Phi(x) \cdot g$ for all $x$ and $g$, and

(b) $p_{Y} \circ \Phi(x)=p(x)$ for all $x$.

Proof. We may assume $X=[0,1]$. Define $\Phi(x)=\left(p(x), f_{x}\right)$ where $f_{x}(g)=x \cdot g$. $\Phi$ has the desired properties. Q.E.D.

The inducing construction is contained in the proof of the following theorem.

THEOREM 1.2. Let a be an action of the strict $G$ space $(Y, \nu)$ on $(S, m)$. Then there exists a Borel mapping $(s, y, g) \mapsto s \cdot(y, g)$ satisfying

(a) $s \cdot(y, e)=$ s a.e. $s$,

(b) $s \cdot\left(y, g_{1}\right) \cdot\left(y g_{1}, g_{2}\right)=s \cdot\left(y, g_{1} g_{2}\right)$ a.e. $s$ for each $\left(y, g_{1}, g_{2}\right)$,

(c) $s \cdot(y, g)=a(y, g)^{-1} s$ a.e. $s$ a.e. $(y, g)$,

(d) $s \mapsto s \cdot(y, g) \in \mathscr{I}(S, m)$ for all $(y, g)$.

Furthermore there exists a strict $G$ action on $(Y \times S, \nu \times m)$ and a $G$ equivariant map $p: Y \times S \rightarrow Y$ satisfying $(y, s) \cdot g=(y \cdot g, s \cdot(y, g))$ a.e. $s$ for each $(y, g)$ and $p(y, s)=y$ a.e. $s$ for each $y$. This construction sets up a one-to-one correspondence between the isomorphism classes of $Y \times G$ actions on $(S, m)$ and the isomorphism classes of extensions over $Y$ of strict $G$ actions on $(Y \times S, \nu \times m)$.

REMARK. A strict $G$ action on $Y \times S$ satisfying $(y, s) \cdot g=(y \cdot g, s \cdot(y, g))$ a.e. $s$ for each $(y, g)$ where $a(y, g) s=s \cdot(y, g)$ a.e. $s$ a.e. $(y, g)$ is said to be induced from $a$.

Proof. We may assume $S=[0,1]$. By [6], there is a strict Borel $G$ action on $(Y \times S, \nu \times m)$ satisfying $(y, s) \cdot g=\left(y \cdot g, a(y, g)^{-1} s\right)$ a.e. $s$ a.e. $(y, g)$. Let $y \cdot(s, g)$ and $s \cdot(y, g)$ be the first and second components of $(y, s) \cdot g$. Set $F=\{(y, g):(y, s) \cdot g=(y \cdot g, s \cdot(y, g))$ a.e. $s$ and $s \mapsto s \cdot(y, g) \in \mathscr{I}(S, m)\}$. 
$F$ is a conull Borel subset of $Y \times G$. Furthermore if $(y, g) \in F$ and $\left(y g, g^{\prime}\right) \in F$, then $\left(y, g g^{\prime}\right) \in F$. Hence $F$ is multiplicative. By Lemma 5.2 of [13], there is a conull Borel set $Y_{0}$ in $Y$ such that if $y \in Y_{0}$ and $y \cdot g \in Y_{0}$, then $(y, g) \in F$. By Lemma A.4 of the appendix we may assume $\left[Y_{0}\right]=Y_{0} \cdot G$ is a Borel set and there exists a Borel map $\theta:\left[Y_{0}\right] \rightarrow G$ satisfying $y \cdot \theta(y)^{-1} \in Y_{0}$ and $\theta(y)=e$ for $y \in Y_{0}$.

Define $s *(y, g)=s$ for $y \notin\left[Y_{0}\right]$ and $s *(y, g)=s \cdot\left(y \theta(y)^{-1}, \theta(y) g \theta(y g)^{-1}\right)$ for $y \in\left[Y_{0}\right]$. Clearly $s *(y, e)=s$ for all $(y, s)$. Furthermore (c) is clear as is (d) for $\left(y \theta(y)^{-1}, \theta(y) g \theta(y g)^{-1}\right) \in F$. Also if $y \in\left[Y_{0}\right]$ then $\left(y \theta(y)^{-1}, \theta(y) g_{1} \theta\left(y g_{1}\right)^{-1}\right)$ and $\left(y g_{1} \theta\left(y g_{1}\right)^{-1}, \theta\left(y g_{1}\right) g_{2} \theta\left(y g_{1} g_{2}\right)^{-1}\right) \in F$. Thus

$$
\begin{aligned}
s & \mapsto s \cdot\left(y \theta(y)^{-1}, \theta(y) g_{1} \theta\left(y g_{1}\right)^{-1}\right) \in \mathscr{I}(S, m), \\
\left(y \theta(y)^{-1}, s\right) \cdot \theta(y) g_{1} \theta\left(y g_{1}\right)^{-1} & =\left(y g_{1} \theta\left(y g_{1}\right)^{-1}, s \cdot\left(y \theta(y)^{-1}, \theta(y) g_{1} \theta\left(y g_{1}\right)^{-1}\right)\right) \text { a.e. } s,
\end{aligned}
$$

and

$$
\begin{aligned}
& \left(y g_{1} \theta\left(y g_{1}\right)^{-1}, s\right) \cdot \theta\left(y g_{1}\right) g_{2} \theta\left(y g_{1} g_{2}\right)^{-1} \\
& \quad=\left(y g_{1} g_{2} \theta\left(y g_{1} g_{2}\right)^{-1}, s \cdot\left(y g_{1} \theta\left(y g_{1}\right)^{-1}, \theta\left(y g_{1}\right) g_{2} \theta\left(y g_{1} g_{2}\right)^{-1}\right)\right) \text { a.e. } s .
\end{aligned}
$$

It follows that for a.e. $s$

$$
s *\left(y, g_{1}\right) *\left(y g_{1}, g_{2}\right)=s *\left(y, g_{1} g_{2}\right)
$$

This gives (b).

To construct the needed strict $G$ action on $Y \times S$, first let

$$
W=\left\{(y, s): s *(y, g) *\left(y g, g^{-1}\right)=s \text { a.e. } g\right\} .
$$

$W$ is clearly $\nu \times m$ conull. Furthermore $W_{y}=\{s:(y, s) \in W\}$ is conull in $S$ for each $y$. Indeed, by (a) and (b), $s *(y, g) *\left(y g, g^{-1}\right)=s$ a.e. $(s, g)$. Thus $s *(y, g) *\left(y g, g^{-1}\right)=s$ a.e. $g$ for a.e. $s$.

Define $\Phi: W \rightarrow Y \times U^{G}$ by $\Phi(y, s)=\left(y, f_{y, s}\right)$ where $f_{y, s}(g)=s *(y, g) . \Phi$ is one-to-one for if $\left(y, f_{y, s}\right)=\left(y^{\prime}, f_{y^{\prime}, s^{\prime}}\right), y=y^{\prime}$ and $s *(y, g)=s^{\prime} *(y, g)$ a.e. $g$. Thus,

$$
s *(y, g) *\left(y g, g^{-1}\right)=s^{\prime} *(y, g) *\left(y g, g^{-1}\right) \text { a.e. } g \text {. }
$$

Thus since $(y, s)$ and $\left(y, s^{\prime}\right) \in W, s=s^{\prime} . \Phi$ is clearly Borel. Hence $\Phi$ extends to an isomorphism of $Y \times S$ onto $Y \times U^{G}$. One can obtain surjectivity if $Y \times S-W$ and $Y \times U^{G}-\Phi(W)$ have the same cardinality. This can be insured by making minor adjustments in $\Phi$ and $W$. Define $(y, s) \cdot g=\Phi^{-1}(\Phi(y, s) \cdot g)$ and define $p$ by $p(y, s)=p_{Y} \circ \Phi(y, s)$. Since $W_{y}$ is conull for each $y, p(y, s)=y$ a.e. $s$ for each $y$. Redefine $s \cdot(y, g)$ by $(y, s) \cdot g=(y \cdot(s, g), s \cdot(y, g))$. Clearly $p(y, s)=y$ a.e. $s$ for each $y$. To show this completes the construction, it suffices to show

$$
s \cdot(y, g)=s *(y, g) \text { a.e. } s \text { for each }(y, g) .
$$


But

$$
(y, s) \cdot g=\Phi^{-1}\left(\left(y, f_{y, s}\right) \cdot g\right)=\Phi^{-1}\left(y \cdot g, f_{y g, s *(y, g)}\right)=(y \cdot g, s *(y, g))
$$

provided $f_{y, s} \cdot g=f_{y \cdot g, s *(y, g)}$ and $(y \cdot g, s *(y, g)) \in W$. Since $(y \cdot g, s *(y, g)) \in$ $W$ a.e. $s$ for each $(y, g)$, we need only note $f_{y, s} \cdot g=f_{y \cdot g, s *(y, g)}$ a.e. $s$. But this is equivalent to $s *\left(y, g g^{\prime}\right)=s *(y, g) *\left(y g, g^{\prime}\right)$ a.e. $\left(s, g^{\prime}\right)$ which follows by (b).

The last statement of the theorem follows from the fact that any isomorphism $\Phi$ over $Y$ between two $G$ actions on $Y \times S$ has form $\Phi(y, s)=(y, B(y)(s))$ a.e. $s$ a.e. $y$, where $B: Y \rightarrow \mathscr{I}(S, m)$ is Borel. This $B$ then institutes the cohomology between the two actions of $Y \times G$. Q.E.D.

If $a$ is an action of $Y \times G$ on $(S, m)$, we shall call it strict if there exists a Borel $\operatorname{map}(s,(y, g)) \rightarrow s \cdot(y, g)$ satisfying (a), (b), and $s \cdot(y, g)=a(y, g)^{-1} s$ a.e. $s$ for each $(y, g)$. Theorem 1.2 shows each action $a$ is equal to a strict action a.e. $(y, g)$. Furthermore, the proof shows if $a$ is a strict action, then there exists a strict $G$ action on $Y \times S$ and a $G$ equivariant Borel map $p: Y \times S \rightarrow Y$ satisfying $(y, s) \cdot g$ $=\left(y \cdot g, a(y, g)^{-1} s\right)$ a.e. $s$ for each $(y, g)$ and $p(y, s)=y$ a.e. $s$ for each $y$. If $a$ is strict, an action induced by $a$ is assumed to satisfy $(y, s) \cdot g=\left(y \cdot g, a(y, g)^{-1} s\right)$ a.e. $s$ for each $(y, g)$.

The following is Proposition 1 of [2].

THEOREM 1.3. Suppose $(Y, \nu)$ is an ergodic $G$ space. Then every extension $X$ of $Y$ is isomorphic over $Y$ to a $G$ space induced by an action of $Y \times G$.

Our correspondence between actions $a$ of $Y \times G$ and extensions of $Y$ in the case when the $G$ action on $(Y, \nu)$ is ergodic can be used to show the equivalence of these notions to that of an action of the measured groupoid $Y \times G$ given in [15]. Indeed, an action with quasi-invariant measure in [15] is essentially an extension of the $G$-space $(Y, \nu)$. If $Y$ is not an ergodic $G$ space, then an action of $Y \times G$ in the sense of [15] would correspond to a disjoint countable direct sum of extensions that are induced from actions of $Y_{i} \times G$ where $Y_{i}$ are disjoint invariant Borel subsets of $Y$ with positive measures.

Let $H$ be a closed subgroup of $G$. The measure groupoid $H \backslash G \times G$ 'defines' a virtual subgroup of $G$ corresponding to the actual subgroup $H$. The following result is not unexpected and its proof follows the ideas used in the argument for Theorem 6.13 of $[15]$.

Proposition 1.4. There is a one-to-one correspondence between the isomorphism classes of $H \backslash G \times G$ actions on $(S, m)$ and the isomorphism classes of $H$ actions on $(S, m)$.

Proof. Let $\gamma$ be a Borel selection on the coset space $H \backslash G$ satisfying $\gamma(H)=e$. Define $J$ mapping $H \backslash G \times G$ to $H$ by $J(x, g)=\gamma(x) g \gamma(x \cdot g)^{-1}$. Then $J$ is a cocycle on $H \backslash G \times G$. Suppose $\phi$ is an action of $H$ on $(S, m)$. Then $\phi \circ J$ is an action of $H \backslash G \times G$ on $(S, m)$. Suppose $\psi$ is an action of $H \backslash G \times G$ on $(S, m)$; 
then $\tilde{\psi}(h) \equiv \psi(H, h)$ is an action of $H$ on $(S, m)$. Clearly $(\phi \circ J)^{\sim}=\phi$. Hence the map $\psi \rightarrow \tilde{\psi}$ is onto. The result will follow if we show $\tilde{\psi} \circ J$ is isomorphic to $\psi$ for any action $\psi$, and if we show $\phi \circ J$ and $\phi^{\prime} \circ J$ are isomorphic as $H \backslash G \times G$ actions iff $\phi$ and $\phi^{\prime}$ are isomorphic $H$ actions.

Note

$$
\begin{aligned}
(\tilde{\psi} \circ J)(x, g) & =\tilde{\psi}\left(\gamma(x) g \gamma(x \cdot g)^{-1}\right)=\psi\left(H, \gamma(x) g \gamma(x \cdot g)^{-1}\right) \\
& =\psi(H, \gamma(x)) \psi(x, g) \psi\left(H, \gamma(x \cdot g)^{-1}\right) \\
& =A(x) \psi(x, g) A(x \cdot g)^{-1}
\end{aligned}
$$

where $A(x)=\psi(H, \gamma(x))$. This shows the first statement.

Now $\phi \circ J$ and $\phi^{\prime} \circ J$ are isomorphic actions iff there is a Borel function $A$ on $H \backslash G$ with values in $\mathscr{I}(S, m)$ satisfying

$$
A(x) \phi\left(\gamma(x) g \gamma(x \cdot g)^{-1}\right)=\phi^{\prime}\left(\gamma(x) g \gamma(x \cdot g)^{-1}\right) A(x \cdot g) \text { a.e. }(x, g) \text {. }
$$

Let $F$ be the set of $(x, g)$ for which the above equation holds. $F$ is conull and multiplicatively closed. By Lemma 5.2 of [13], there is a conull Borel subset $U \subseteq H \backslash G$ such that if $x$ and $x \cdot g$ belong to $U$, then $(x, g) \in F$. Choose $x_{0} \in U$. Then $x_{0} \gamma\left(x_{0}\right)^{-1} h \gamma\left(x_{0}\right) \in U$ for each $h \in H$. Hence

$$
\begin{aligned}
& A\left(x_{0}\right) \phi\left(\gamma\left(x_{0}\right) \gamma\left(x_{0}\right)^{-1} h \gamma\left(x_{0}\right) \gamma\left(x_{0} \cdot \gamma\left(x_{0}\right)^{-1} h \gamma\left(x_{0}\right)\right)^{-1}\right) \\
& \quad=\phi^{\prime}\left(\gamma\left(x_{0}\right) \gamma\left(x_{0}\right)^{-1} h \gamma\left(x_{0}\right) \gamma\left(x_{0} \cdot \gamma\left(x_{0}\right)^{-1} h \gamma\left(x_{0}\right)\right)^{-1}\right) A\left(x_{0} \cdot \gamma\left(x_{0}\right)^{-1} h \gamma\left(x_{0}\right)\right) .
\end{aligned}
$$

Hence $A\left(x_{0}\right) \phi(h)=\phi^{\prime}(h) A\left(x_{0}\right)$ for all $h$. Thus $\phi$ and $\phi^{\prime}$ are isomorphic. If $A \phi(h)=\phi^{\prime}(h) A$ for all $h$, then

$$
A \phi\left(\gamma(x) g \gamma(x \cdot g)^{-1}\right)=\phi^{\prime}\left(\gamma(x) g \gamma(x \cdot g)^{-1}\right) A \text { for all }(x, g) \text {. }
$$

Thus $\phi \circ J$ is isomorphic to $\phi^{\prime} \circ J$. Q.E.D.

COROllary 1.5 (ZIMmer). If $(X, \mu)$ is an extension of $H \backslash G$, then $X$ is isomorphic over $H \backslash G$ to $H \backslash G \times S$ where $(S, m)$ is an $H$ space and the action of $G$ on $H \backslash G \times S$ is defined by $(x, s) \cdot g=\left(x \cdot g, s \cdot \gamma(x) g \gamma(x \cdot g)^{-1}\right)$.

Definition 1.6. Suppose $(Y, \nu)$ is an ergodic $G$ space. A $Y \times G$ action $a$ on $(S, m)$ is ergodic if whenever $y \rightarrow E_{y}$ is a Borel map from $Y$ into the measure algebra $M(S, m)$ defined by $m$ satisfying $E_{y \cdot g}=a(y, g)^{-1} E_{y}$ a.e. $(y, g)$, then $E_{y}$ is null a.e. $y$ or $E_{y}$ is conull a.e. $y$.

Proposition 1.7. A $Y \times H$ action a on $(S, m)$ is ergodic iff the action it induces on $(Y \times S, \nu \times m)$ is ergodic.

Proof. Note that there is a one-to-one correspondence between the set of $G$ invariant Borel sets $E$ of $Y \times S$ and Borel maps $y \mapsto E_{y} \in M(S, m)$ satisfying $E_{y \cdot g}=a(y, g)^{-1} E_{y}$ a.e. $(y, g)$ given by $E_{y}=\{s:(y, s) \in E\}$. Q.E.D. 
Proposition 1.8. The correspondence between $H \backslash G \times G$ actions and $H$ actions given in Proposition 1.3 preserves ergodicity.

Proof. Let $\psi: H \rightarrow \mathscr{I}(S, m)$ be an action of $H$ on $(S, m)$. Then $a(x, g)=$ $\psi\left(\gamma(x) g \gamma(x \cdot g)^{-1}\right)$ defines the corresponding action on $H \backslash G \times G$. Suppose $\psi$ is ergodic and $x \mapsto E_{x} \in M(S, m)$ is Borel and satisfies $E_{x \cdot g}=a(x, g)^{-1} E_{x}$ a.e. By Proposition A.5 of the appendix one may redefine $E_{x}$ on a set of measure 0 so that $E_{x \cdot g}=a(x, g)^{-1} E_{x}$ for all $(x, g)$. Thus $E_{x_{0} \cdot h}=\psi(h)^{-1} E_{x_{0}}$ for all $h$, where $x_{0}=H$. By ergodicity of $\psi, E_{x_{0}}$ is null or conull. Thus $E_{x_{0} g}=a\left(x_{0}, g\right)^{-1} E_{x_{0}}$ is everywhere null or everywhere conull. The converse is even easier. Q.E.D.

Corollary 1.9 (ZIMmer). Let $(S, m)$ be an $H$ space. Then the induced $G$ space $H \backslash G \times S$ is $G$ ergodic iff $S$ is an ergodic $H$ space.

Let $\psi: G \rightarrow \mathscr{I}(S, m)$ give an action of $G$ on $(S, m)$. Then if $(Y, \nu)$ is an ergodic $G$ space, the action $a$ on $Y \times G$ defined by $a(y, g)=\psi(g)$ is called the restriction of the $G$ action $\psi$ to $Y \times G$.

Proposition 1.10. Let $G$ act on $(S, m)$. Let $H$ be a closed subgroup of $G$. Then the restriction of the $G$ action to $H \backslash G \times G$ is isomorphic to the $H \backslash G \times G$ action on $S$ given by the $H$ action on $S$.

Proof. $a(y, g)=\psi(g)$. Define $A(y) \in \mathscr{I}(S, m)$ by $A(y)=\psi(\gamma(y))$ for $y \in$ $H \backslash G$. Then

$$
\begin{aligned}
A(y) a(y, g) A(y \cdot g)^{-1} & =\psi(\gamma(y)) \psi(g) \psi(\gamma(y \cdot g))^{-1} \\
& =\psi\left(\gamma(y) g \gamma(y \cdot g)^{-1}\right) . \quad \text { Q.E.D. }
\end{aligned}
$$

COROllary 1.11 (MACKey-Moore-ZIMmer). Let $G$ act ergodically on $(S, m)$. Then $H$ acts ergodically on $S$ iff the restriction of the $G$ action to $H \backslash G \times G$ acts ergodically on $S$ iff the product $G$ action on $H \backslash G \times S$ is ergodic.

Our next topics of discussion involve the concept of a homomorphism between ergodic group actions and its Mackey range. The former is the concept of a group homomorphism in the virtual subgroup setting. Its definition is taken from [13].

A homomorphism between a strict ergodic $G$ space $(X, \mu)$ and a strict ergodic $K$ space $(Y, \nu)$ is a pair of Borel maps $h=(p, \phi), p: X \rightarrow Y, \phi: X \times G \rightarrow K$ satisfying

(1.6) $\phi$ is a cocycle,

(1.7) $p(x \cdot g)=p(x) \cdot \phi(x, g)$ for all $(x, g)$, and

(1.8) if $E$ is a strictly invariant analytic subset of $Y, \mu\left(p^{-1}(E)\right)=0$ iff $\nu(E)=0$. We note that (1.6) and (1.7) just say that $h: X \times G \rightarrow Y \times K$ is multiplicative between these two groupoids.

The Mackey range of $h$ is the concept of the closure of the range of a homomorphism in the virtual group setting. The construction of the Mackey range involves 
the disintegration of a nonergodic $G$ space $(X, \mu)$ into ergodic components. Specifically, if $(X, \mu)$ is a nonergodic $G$ space, then there exists a standard Borel space $T$ and a Borel map $F: X \rightarrow T$ such that if $\mu=\int \mu_{t} d F_{*} \mu(t)$ is the disintegration of $\mu$ over the fibers of $F$, then a.e. $\left(X, \mu_{t}\right)$ is an ergodic $G$ space. Furthermore, $F$ enjoys the following universal property. If $h$ is a Borel function on $X$ with values in a countably separated Borel space and if $h$ is essentially invariant, i.e. $h(x \cdot g)=h(x)$ a.e. $(x, g)$, then there exists an essentially unique Borel function $H$ on $T$ such that $H \circ F(x)=h(x)$ a.e. $x$. This universal property insures the essential uniqueness of the ergodic decomposition $(F, T)$.

Suppose $(X, \mu)$ is a strict ergodic $G$ space and $(Y, \nu)$ is a strict ergodic $K$ space. Suppose $h=(p, \phi)$ is a homomorphism from $X \times G$ into $Y \times K$. In the appendix we show $h$ may be redefined on a set of measure 0 so that the following hold.

(1.9) $h=(p, \phi)$ is a homomorphism from $X \times G$ to $Y \times K$,

(1.10) there is an ergodic extension $\sigma_{h}:\left(Y_{h}, \nu_{h}\right) \rightarrow(Y, \nu)$ and a homomorphism $h^{\prime}=\left(p^{\prime}, \phi\right): X \times G \rightarrow Y_{h} \times K$ such that $i \circ h^{\prime}(x, g)=h(x, g)$ for all $(x, g)$, where $i(y, k)=\left(\sigma_{h}(y), k\right)$ is the inclusion homomorphism of $Y_{h} \times K$ into $Y \times K$, and

(1.11) the mapping $(x, k) \mapsto p^{\prime}(x) k^{-1}$ is an ergodic decomposition of the $G$ action on $X \times K$ defined by $(x, k) \cdot g=(x \cdot g, k \phi(x, g))$.

The $K$ space $\left(Y_{h}, \nu_{h}\right)$ is called the Mackey range of $h$. If $\sigma_{h}$ is an isomorphism of $Y_{h}$ onto $Y$, the homomorphism $h$ is said to have dense range. In this case, we can just as well take $h^{\prime}=h$ and $Y_{h}=Y$. In particular, the map $(x, k) \mapsto p(x) k^{-1}$ gives the ergodic decomposition of $X \times K$.

Suppose $h: X \times G \rightarrow Y \times K$ is a homomorphism between the strict $G$ space $(X, \mu)$ and the strict $K$ space $(Y, \nu)$. Suppose $a: Y \times K \rightarrow \mathscr{I}(S, m)$ defines a strict action of $Y \times K$ on $(S, m)$. Then $h^{*} a=a \circ h$ defines an action of $X \times G$ called the pull back of the action $a$ under $h$. The isomorphically unique $G$ action on $X \times S$ induced by $h^{*} a$ is denoted by $X \times_{h} S$ and is called the fibered skew product extension of $X$ over $h$ with the $K$ space induced by $a$.

There is an alternate definition of a fibered skew product extension which is equivalent to the one just given in which the action is skew and defined over a fibered product.

In fact, suppose $h=(p, \phi)$ is a homomorphism from the ergodic $G$ space $(X, \mu)$ into the ergodic $K$ space $(Y, \nu)$. Suppose $q:(W, \lambda) \rightarrow(Y, \nu)$ is an extension of the $K$ space $(Y, \nu)$. Define $X \times{ }_{h} W=\{(x, w): p(x)=q(w)\}$ with action defined by $(x, w) \cdot g=(x \cdot g, w \cdot \phi(x, g))$. This action is well defined by property (1.7). To obtain the needed measure class on $X \times{ }_{h} W$, one first disintegrates the measure $\lambda$ over the fibers of $q$, i.e. $\lambda=\int^{\oplus} \lambda_{y} d \nu(y)$. Since $\lambda \cdot k(E) \equiv \lambda\left(E \cdot k^{-1}\right)$ and $(\nu \cdot k)(E)=\nu\left(E \cdot k^{-1}\right)$ define measures equivalent to $\lambda$ and $\nu$, respectively, for each $k$, it follows that $\lambda_{y \cdot k} \sim \lambda_{y} \cdot k \nu$ a.e. $y$ for each $k$. By Proposition A.6 $\lambda_{y}$ may be redefined on a set of measure 0 so that $\lambda_{y \cdot k} \sim \lambda_{y} \cdot k$ for all $(y, k)$. Define $\mu \times{ }_{h} \lambda=\int^{\oplus} \varepsilon_{x} \times \lambda_{p x} d \mu(x)$ where $\varepsilon_{x}$ is the point mass at $x$. We show this measure's measure class is well defined and quasi-invariant. To see that it is well defined we show that if $\lambda=\int \lambda_{y}^{\prime} d \nu(y)$ is another decomposition of $\lambda$ over $q$ and $\lambda_{y \cdot k}^{\prime} \sim \lambda_{y}^{\prime} \cdot k$ for all $(y, k)$, then $\lambda_{p x} \sim \lambda_{p x}^{\prime} \mu$ a.e. $x$. Indeed, set $Y_{0}=\left\{y: \lambda_{y}^{\prime} \sim \lambda_{y}\right\}$. $Y_{0}$ is a strictly invariant conull Borel subset of $Y$. By (1.8), $p^{-1}\left(Y_{0}\right)$ is conull. Thus 
$\lambda_{p x} \sim \lambda_{p x}^{\prime} \mu$ a.e. $x$. Hence $\left[\mu \times_{h} \lambda\right]$ is well defined. To see that $\mu \times_{h} \lambda$ is quasiinvariant, note

$$
\begin{aligned}
\left(\mu \times_{h} \lambda\right) \cdot g & =\int^{\oplus}\left(\varepsilon_{x} \times \lambda_{p x}\right) \cdot g d \mu(x) \\
& =\int^{\oplus} \varepsilon_{x \cdot g} \times \lambda_{p x} \cdot \phi(x, g) d \mu(x) \\
& \sim \int^{\oplus} \varepsilon_{x \cdot g} \times \lambda_{p(x \cdot g)} d \mu(x) \\
& \sim \int^{\oplus} \varepsilon_{x} \times \lambda_{p x} d \mu(x)=\mu \times_{h} \lambda .
\end{aligned}
$$

To see these definitions of fibered skew product extensions are equivalent, by Theorems 1.2 and 1.3 we may suppose $W$ is induced by a strict action $a$ of $Y \times K$ on $(S, m)$. Thus $W=Y \times S, \lambda=\nu \times m$, and $(y, s) \cdot k=(y \cdot k, s \cdot(y, k))$ a.e. $s$ for each $(y, k)$. Also since one has $q(y, s)=y$ a.e. $s$ for each $y$ and $s \mapsto s \cdot(y, k)$ is in $\mathscr{I}(S, m)$ for each $(y, k)$, we see that $\lambda=\int^{\oplus} \varepsilon_{y} \times m d \nu(y)$ and $\left(\varepsilon_{y} \times m\right) \cdot k \sim$ $\varepsilon_{y \cdot k} \times m$ for all $(y, k)$. Thus $X \times{ }_{h} W=\{(x, y, s): p x=q(y, s)\}$ with measure $\mu \times{ }_{h} \lambda=\int \varepsilon_{x} \times\left(\varepsilon_{p x} \times m\right) d \mu(x)$ is isomorphic to $X \times S$ with measure $\mu \times m$; the isomorphism sending $(x, y, s) \mapsto(x, s)$. Under this isomorphism, the action on $X \times{ }_{h} W$ corresponds to the action on $X \times S$ given by $(x, s) \cdot g=(x \cdot g, s \cdot h(x, g))$ which is clearly the action induced by $h^{*} a$ since

$$
\begin{aligned}
\left(h^{*} a\right)(x, g)^{-1} s & =a(p(x), \phi(x, g))^{-1} s \\
& =s \cdot(p(x), \phi(x, g))=s \cdot h(x, g) \text { a.e. } s .
\end{aligned}
$$

Our next result determines when the pull back of an action is ergodic.

Proposition 1.12. Let $(X, \mu)$ be an ergodic $G$ space and $(Y, \nu)$ be an ergodic $K$ space. Let $h: X \times G \rightarrow Y \times K$ be a homomorphism. Suppose $a$ is an action of $Y \times K$ on $(S, m)$. Then $h^{*} a$ is an ergodic action of $X \times G$ iff the restriction of a to $Y_{h} \times K$ is ergodic.

Proof. Suppose the restriction of $a$ to $Y_{h} \times K$ is ergodic. Let $x \mapsto E_{x}$ be a Borel map of $X$ into $M(S, m)$ satisfying

$$
E_{x \cdot g}=h^{*} a(x, g)^{-1} E_{x} \quad \text { a.e. }(x, g) .
$$

Define $F(x, k)=a\left(p(x) k^{-1}, k\right) E_{x}$. Then $F$ is a Borel map of $X \times K$ into $M(S, m)$ and

$$
\begin{aligned}
F(x \cdot g, k \phi(x, g)) & =a\left(p(x \cdot g) \phi(x, g)^{-1} k^{-1}, k \phi(x, g)\right) E_{x \cdot g} \\
& =a\left(p(x) k^{-1}, k \phi(x, g)\right) a(p(x), \phi(x, g))^{-1} E_{x} \\
& =a\left(p(x) k^{-1}, k\right) E_{x} \\
& =F(x, k) \quad \text { a.e. }(x, k, g) .
\end{aligned}
$$


Since $(x, k) \mapsto p_{h}(x) k^{-1}$ is the ergodic decomposition of the $G$ space $X \times K$ and $F$ is $G$ invariant, there exists a Borel function $W$ on $Y_{h}$ such that $W\left(p_{h}(x) k^{-1}\right)=$ $F(x, k)$ a.e. $(x, k)$. Thus

$$
\begin{aligned}
W\left(p_{h}(x) k^{-1} k_{1}\right) & =F\left(x, k_{1}^{-1} k\right)=a\left(p(x) k^{-1} k_{1}, k_{1}^{-1} k\right) E_{x} \\
& =a\left(p(x) k^{-1} k_{1}, k_{1}^{-1}\right) a\left(p(x) k^{-1}, k\right) E_{x} \\
& =a\left(p(x) k^{-1}, k_{1}\right)^{-1} F(x, k) \\
& =a\left(p(x) k^{-1}, k_{1}\right)^{-1} W\left(p_{h}(x) k^{-1}\right)
\end{aligned}
$$

a.e. $(x, k)$ for each $k_{1}$. Since the collection of all $p_{h}(x) k^{-1}$ is conull in $Y_{h}$, one has

$$
W\left(y \cdot k_{1}\right)=a\left(y, k_{1}\right)^{-1} W(y) \text { a.e. }\left(y, k_{1}\right) \in Y_{h} \times K \text {. }
$$

Since $a$ restricted to $Y_{h} \times K$ is ergodic, $W(y)$ is a.e. conull or a.e. null. Thus $E_{x}$ is a.e. conull or a.e. null. Thus $h^{*} a$ is an ergodic action.

Conversely, suppose that $h^{*} a=a \circ h$ is an ergodic action of $X \times G$. Let $y \mapsto$ $W(y)$ be a Borel mapping of $Y_{h}$ into $M(S, m)$ satisfying $W(y \cdot k)=a(y, k)^{-1} W(y)$ a.e. $(y, k)$. By Proposition A.5 of the appendix, we may assume this holds for all $y$ and $k$. Set $E_{x}=W\left(p_{h}(x)\right)$. Then

$$
\begin{aligned}
E_{x \cdot g} & =W\left(p_{h}(x \cdot g)\right)=W\left(p_{h}(x) \cdot \phi(x, g)\right) \\
& =a\left(p_{h}(x), \phi(x, g)\right)^{-1} E_{x} \\
& =h^{*} a(x, g) E_{x} \text { for all }(x, g) .
\end{aligned}
$$

Since $h^{*} a$ is ergodic, $E_{x}$ is null a.e. $x$ or $E_{x}$ is conull a.e. $x$. Thus

$$
\begin{aligned}
W\left(p_{h}(x) k^{-1}\right) & =a\left(p_{h}(x), k^{-1}\right)^{-1} W\left(p_{h}(x)\right) \\
& =a\left(p_{h}(x), k^{-1}\right)^{-1} E_{x}
\end{aligned}
$$

is a.e. null or a.e. conull on $X \times K$. Thus $W(y)$ is a.e. null or a.e. conull. Q.E.D.

Corollary 1.13. Let a be a strict action of $Y \times K$ on $(S, m)$. Let $h$ be a homomorphism from $X \times G$ into $Y \times K$. Then the fibered skew product $G$ space $X \times_{h} S$ is ergodic iff the restriction of a to the Mackey range of $h$ is ergodic.

Corollary 1.14. Let $q:(W, \lambda) \rightarrow(Y, \nu)$ be an extension of $(Y, \nu)$. Let $h$ be a homomorphism from $X \times G$ into $Y \times K$. Then the fibered skew product space $X \times{ }_{h} W$ is ergodic iff the fibered product space $Y_{h} \times_{\left(\sigma_{h, i}\right)} W$ is an ergodic $K$ space.

COROllary 1.15 (ZIMMER). Let $\phi: X \times G \rightarrow H$ be a cocycle with Mackey range the $H$ space $(Y, \nu)$. Let $S$ be an $H$ space. Then $X \times_{\phi} S$ is an ergodic $G$ space iff $Y \times S$ is an ergodic $H$ space.

Let $(Y, \nu)$ be an ergodic $K$ space. By a cocycle representation of $Y \times K$ on a separable Hilbert space $\mathscr{H}$, one means a cocycle $L$ on $Y \times K$ with values in the unitary group of $\mathscr{H}$. If $L_{1}$ and $L_{2}$ are cocycle representations on $\mathscr{H}_{1}$ and $\mathscr{H}_{2}$, they are said to be equivalent if there exists a strongly Borel function $A$ from $Y$ into the set of unitary transformations from $\mathscr{H}_{1}$ onto $\mathscr{H}_{2}$ satisfying $A(y) L_{1}(y, k)=$ $L_{2}(y, k) A(y \cdot k)$ a.e. $(y, k)$. A representation $L$ on $Y \times K$ is said to be irreducible 
if whenever $y \mapsto A(y)$ is a strongly Borel map from $Y$ into the bounded linear operators on $\mathscr{H}$ and $A(y) L(y, k)=L(y, k) A(y \cdot k)$ a.e. $(y, k)$, then $A(y)=c I$ a.e. $y$ for some constant $c$. This is equivalent to the following. Whenever $y \mapsto E(y)$ is a strongly Borel orthogonal projection valued function satisfying $E(y) L(y, k)=$ $L(y, k) E(y \cdot k)$ a.e. $(y, k)$, then $E(y)=0$ a.e. $y$ or $E(y)=I$ a.e. $y$. That is there is no equivariant Borel field of proper closed subspaces.

The notion of a bundle representation of $Y \times K$ is similar to that of a cocycle representation except that it is defined over a Hilbert bundle over $Y$. Namely, let $y \rightarrow \mathscr{H}_{y}$ be an Hilbert bundle over $Y$. A bundle representation is then a Borel map $(y, k) \mapsto L(y, k)$, where $L(y, k)$ is a unitary transformation of $\mathscr{H}_{y \cdot k}$ onto $\mathscr{H}_{y}$ and $L\left(y, k_{1}\right) L\left(y k_{1}, k_{2}\right)=L\left(y, k_{1} k_{1}\right)$ a.e. $\left(y, k_{1}, k_{2}\right)$. The definitions for equivalence and irreducibility are the same as for cocycle representations except that the maps $y \mapsto A(y)$ are now Borel fields of bounded operators between the bundles. Moreover, since the action of $K$ on $(Y, \nu)$ is ergodic and every Hilbert bundle can be measurably trivialized, every bundle representation is equivalent to a cocycle representation.

Suppose $(X, \mu)$ is an ergodic $G$ space and $h$ is a homomorphism from $X \times G$ into $Y \times K$. Then $h$ induces a correspondence from the bundle representations of $Y \times K$ into those for $X \times G$. Namely, if $L$ is a bundle representation of $Y \times K$ on the bundle $y \mapsto \mathscr{H}_{y}$, then $h^{*} L$ is the bundle representation of $X \times G$ on the bundle $x \rightarrow \mathscr{H}_{p x}$ defined by $(x, g) \rightarrow L(h(x, g))$. If $H=G$ and $\phi(x, g)=g$, then $p$ : $X \rightarrow Y$ is an extension and $h^{*} L$ is called the restriction of $L$ to $X \times G$. Since bundle representations are equivalent to cocycle representations, we shall in most instances consider only the latter.

Proposition 1.16. Let $L_{1}$ and $L_{2}$ be cocycle representations of $Y \times K$. Then $h^{*} L_{1} \simeq h^{*} L_{2}$ iff the restrictions of $L_{1}$ and $L_{2}$ to the Mackey range of $h$ are equivalent.

Proposition 1.17. Suppose $h$ is a homomorphism from $X \times G$ into $Y \times K$. Let $L$ be an irreducible cocycle representation of $Y \times K$. Then $h^{*} L$ is irreducible iff $L$ restricted to the Mackey range of $h$ is irreducible.

Propositions 1.16 and 1.17 follow from Theorem 7.17 of [15] and Theorem A.10 and Proposition A.11 of the appendix.

Moreover one can modify the proof of Theorem 7.17 to show that if $h: X \times G \rightarrow$ $Y \times K$ has dense range then actions $a_{1}$ and $a_{2}$ of $Y \times K$ are equivalent iff $h^{*} a_{1}$ and $h^{*} a_{2}$ are equivalent actions of $X \times G$.

Many of the results in our next section relate the structure of actions and representations to spectral properties of the representations they induce. Suppose $\pi$ is a Hilbert bundle representation of the virtual subgroup $(X \times G, \mu \times m)$ and $(X \times G, \mu \times m)$ is a subvirtual subgroup of $(Y \times G, \nu \times m)$; i.e. $(X, \mu)$ and $(Y, \nu)$ are ergodic $G$ spaces and $p:(X, \mu) \rightarrow(Y, \nu)$ is an extension of $Y$. Thus $p_{*} \mu \sim \nu$ and if $\mu=\int^{\oplus} \mu_{y} d \nu(y)$ is the disintegration of the measure $\mu$ over the fibers of $p$, one has $\mu_{y \cdot g} \sim \mu_{y} \cdot g$ a.e. $(y, g)$, where $\left(\mu_{y} \cdot g\right)(E)=\mu_{y}\left(E g^{-1}\right)$. By Proposition A.6 we may redefine $y \mapsto \mu_{y}$ on a null set of $Y$ so that $\mu_{y \cdot g} \sim \mu_{y} \cdot g$ for all $(y, g)$. Define 
ind $\pi$ to be the bundle representation on the Hilbert bundle $y \mapsto \mathscr{K}_{y}=\int^{\oplus} \mathscr{H}_{x} d \mu_{y}(x)$ by

$$
\text { (ind } \pi)(y, g) f(x)=\frac{d \mu_{y \cdot g}}{d \mu_{y} \cdot g}(x \cdot g)^{1 / 2} \pi(x, g) f(x \cdot g) \text {. }
$$

Ind $\pi$ is a bundle representation of $Y \times G$ called the bundle representation induced by $\pi$. If $\pi=1$, the trivial representation, ind $\pi$ is called the natural bundle representation of the extension.

2. The structure theorems. In this section we shall give cohomological and representation theoretic characterizations of the actions and representations of $X \times G$ which are pull backs of actions and representations of $Y \times K$ under a homomorphism $h$ from $X \times G$ into $Y \times K$ with dense range. We then use these results to obtain structure theorems for fibered skew product actions.

Suppose $h=(p, \phi)$ is the homomorphism. One can form the fibered skew $G$ space $X \times_{h}(Y \times K)$ which is clearly isomorphic to the skew product $G$ space $X \times{ }_{\phi} K$. The next result is the major tool of this section.

Proposition 2.1. Let $h$ be a homomorphism from the $G$ space $(X, \mu)$ into the $K$ space $(Y, \nu)$ with dense range. Let $b$ be a cocycle on $X \times G$ with values in a complete separable metric group $M$. Then a necessary and sufficient condition for $b$ to be cohomologous to $h^{*}$ a for some cocycle $a$ on $Y \times K$ with values in $M$ is that $b_{\mid X \times_{\phi} K \times G}$ be cohomologous to the identity cocycle.

Proof. Suppose $b=h^{*} a$. Set $A(x, k)=a\left(p(x) k^{-1}, k\right)$. Then

$$
\begin{aligned}
A(x, k) h^{*} a(x, g) & =A(x, k) a(p(x), \phi(x, g)) \\
& =a\left(p(x) k^{-1}, k\right) a(p(x), \phi(x, g))=a\left(p(x) k^{-1}, k \phi(x, g)\right) \\
& =a\left(p(x \cdot g)(k \phi(x, g))^{-1}, k \phi(x, g)\right)=A((x, k) \cdot g) .
\end{aligned}
$$

Thus $h^{*} a$ restricted to $X \times{ }_{\phi} K \times G$ is cohomologous to the identity.

Conversely, suppose $b_{\mid X \times_{\phi} K \times G}$ is cohomologous to the identity. Then there exists a Borel functon $A: X \times K \rightarrow M$ such that

$$
A(x \cdot g, k \phi(x, g))=A(x, k) b(x, g) \text { a.e. }(x, k, g) \text {. }
$$

LEMMA A. There is a Borel function $a: Y \times K \rightarrow M$ satisfying

$$
A\left(x, k_{1}\right) A\left(x, k_{2}^{-1} k_{1}\right)^{-1}=a\left(p(x) k_{1}^{-1}, k_{2}\right) \text { a.e. }\left(x, k_{1}, k_{2}\right) \text {. }
$$

Proof. Set $F\left(x, k_{1}, k_{2}\right)=A\left(x, k_{1}\right) A\left(x, k_{2}^{-1} k_{1}\right)^{-1}$. Then $F$ is a Borel function and $F\left(x \cdot g, k_{1} \phi(x, g), k_{2}\right)=F\left(x, k_{1}, k_{2}\right)$ a.e. $\left(x, k_{1}, k_{2}\right)$ for each $g$. But $\left(x, k_{1}, k_{2}\right)$ $\mapsto\left(p(x) k_{1}^{-1}, k_{2}\right)$ gives the ergodic decomposition of $X \times_{\phi} K \times K$, where the latter has action defined by $\left(x, k_{1}, k_{2}\right) \cdot g=\left(x \cdot g, k_{1} \phi(x, g), k_{2}\right)$. Thus there is a Borel function $a$ on $Y \times K$ satisfying

$$
a\left(p(x) k_{1}^{-1}, k_{2}\right)=A\left(x, k_{1}\right) A\left(x, k_{2}^{-1} k_{1}\right)^{-1} \text { a.e. }\left(x, k_{1}, k_{2}\right) \text {. Q.E.D. }
$$

LEMMA B. $a\left(y, k_{1}\right) a\left(y k_{1}, k_{2}\right)=a\left(y, k_{1} k_{2}\right)$ a.e. $\left(y, k_{1}, k_{2}\right)$. 
ProOF.

$$
\begin{aligned}
& a\left(p(x) k^{-1}, k_{1} k_{2}\right)=A(x, k) A\left(x, k_{2}^{-1} k_{1}^{-1} k\right)^{-1} \\
& =A(x, k) A\left(x, k_{1}^{-1} k\right)^{-1} A\left(x, k_{1}^{-1} k\right) A\left(x, k_{2}^{-1} k_{1}^{-1} k\right)^{-1} \\
& \quad=a\left(p(x) k^{-1}, k_{1}\right) a\left(p(x) k^{-1} k_{1}, k_{2}\right) \text { a.e. }\left(x, k, k_{1}, k_{2}\right) \text {. Q.E.D. }
\end{aligned}
$$

By Corollary A. 8 of the appendix, we may redefine $a$ on a set of measure 0 so that $a$ is strictly multiplicative. Hence we have the following:

$$
a \text { is a cocycle on } Y \times K \text { and }
$$

$$
A\left(x, k_{1}\right) A\left(x, k_{2}^{-1} k_{1}\right)^{-1}=a\left(p(x) k_{1}^{-1}, k_{2}\right) \text { a.e. }\left(x, k_{2}, k_{1}\right) .
$$

LEMMA C. There is a Borel function $B$ from $X$ into $M$ satisfying

$$
A(x, k)=a\left(p(x) k^{-1}, k\right) B(x) \text { a.e. }(x, k) .
$$

Proof. Define $F(x, k)=A(x, k)^{-1} a\left(p(x) k^{-1}, k\right)$. Then $F$ is a Borel function and

$$
\begin{aligned}
F\left(x, h^{-1} k\right) & =A\left(x, h^{-1} k\right)^{-1} a\left(p(x) k^{-1} h, h^{-1} k\right) \\
& =A(x, k)^{-1} a\left(p(x) k^{-1}, h\right) a\left(p(x) k^{-1} h, h^{-1} k\right) \\
& =A\left(x, k^{-1}\right) a\left(p(x) k^{-1}, k\right) .
\end{aligned}
$$

Thus there exists a Borel function $B$ from $X$ into $M$ satisfying $B(x)^{-1}=F(x, k)$ a.e. $(x, k)$. Q.E.D.

We finish the proof. By Lemma $\mathrm{C}$ we have

$$
A(x \cdot g, k \phi(x, g))=a\left(p(x \cdot g) \phi(x, g)^{-1} k^{-1}, k \phi(x, g)\right) B(x \cdot g)
$$

a.e. $(x, k)$ for each $g$. Thus

$$
\begin{aligned}
A(x \cdot g, k \phi(x, g)) & =a\left(p(x) k^{-1}, k\right) a(p(x), \phi(x, g)) B(x \cdot g) \\
& =a\left(p(x) k^{-1}, k\right) B(x) B(x)^{-1} a(p(x), \phi(x, g)) B(x \cdot g) \\
& =A(x, k) B(x)^{-1} a(p(x), \phi(x, g)) B(x \cdot g)
\end{aligned}
$$

a.e. $(x, k)$ for each $g$. But by (2.1), $A(x \cdot g, k \phi(x, g))=A(x, k) b(x, g)$ a.e. $(x, k, g)$. Thus $b(x, g)=B(x)^{-1} a(p(x), \phi(x, g)) B(x \cdot g)$. Hence $b$ is cohomologous to $h^{*} a$. Q.E.D.

Corollary 2.2. Suppose $h: X \times G \rightarrow Y \times K$ is a homomorphism with dense range. Let $\pi$ be a cocycle representation of $X \times G$. Then $\pi \simeq h^{*} \pi^{\prime}$ for some cocycle representation $\pi^{\prime}$ iff $\pi_{\mid X \times{ }_{\phi} K \times G} \simeq I$.

COROllary 2.3. Let $\phi$ be a continuous group homomorphism of $G$ into $K$ with dense range. Let $L$ be a unitary representation of $G$. Then $L=\phi^{*} L^{\prime}$ for some unitary representation $L^{\prime}$ of $K$ iff $L_{\mid K \times G} \simeq I$. 
COROllary 2.4. Let $h: X \times G \rightarrow Y \times K$ be a homomorphism and let $b$ be a cocycle on $X \times G$ with values in $M$. Suppose

$$
\begin{array}{ccc}
X \times G & \stackrel{h}{\rightarrow} & Y \times K \\
\searrow h^{\prime} & & \nearrow \sigma_{h \times i} \\
& Y_{h} \times K &
\end{array}
$$

is the factorization of $h$ through its Mackey range. Then $b$ is cohomologous to $h^{\prime *} a$ for some cocycle $a$ on $Y_{h} \times K$ with values in $M$ iff $b_{\mid X \times{ }_{\phi} K \times G} \simeq I$.

In [17] Caroline Series defined the inverse image of the $K$-space $Y$ under a cocycle $\phi: X \times G \rightarrow K$ having dense range to be the $G$ space induced by $\phi^{*} a$ where $a$ is the action of $K$ on $Y$. If $\phi$ were a homomorphism of $G$ into $K$ and $Y=H \backslash K$ where $H$ is a closed subgroup of $K$, then this induced space would be isomorphic to $\phi^{-1}(H) \backslash G$. The following proposition shows it would be appropriate to call the $G$ space induced by $h^{*} a$ the inverse image under $h$ of the $K$ space induced by the $Y \times K$ action $a$ on $(S, m)$. This proposition is a generalization of Theorem 2.1 of [22] and Proposition 4.11 of [17].

Proposition 2.5. Suppose $h$ is a homomorphism of the ergodic $G$ space $(X, \mu)$ into the ergodic $K$ space $(Y, \nu)$ with dense range. Let a be an ergodic action of $Y \times K$ on $(S, m)$. Suppose $W$ is an extension of $X$ induced by the action $h^{*} a$. Then $h_{\mid W \times G}$ has Mackey range the $K$ space induced by $a$.

Proof. We may assume $W=X \times S$ and $(x, s) \cdot g=\left(x \cdot g, a(p(x), \phi(x, g))^{-1} s\right)$ a.e. $s$ a.e. $(x, g)$.

Let $\tilde{h}=h_{\mid W \times G}$. Then $\tilde{h}(x, s, g)=(p x, \phi(x, g))$ a.e. $(x, g)$. Thus $W \times K$ is isomorphic to $X \times S \times K$ with action given by

$$
(x, s, k) \cdot g=\left(x \cdot g, a(p(x), \phi(x, g))^{-1} s, k \phi(x, g)\right) \text { a.e. } s \text { a.e. }(k, x, g) \text {. }
$$

Let $F$ be a Borel function on $X \times S \times K$ satisfying $F(x, s, k)=$ $\left(p(x) k^{-1}, a\left(p(x) k^{-1}, k\right) s\right)$ a.e. $s$ a.e. $(x, k)$. Note

$$
F\left(x \cdot g, a(p(x), \phi(x, g))^{-1} s, k \phi(x, g)\right)=F(x, s, k) \text { a.e. } s \text { a.e. }(k, x, g) \text {. }
$$

Since $(x, k) \mapsto p(x) k^{-1}$ is an ergodic decomposition of $X \times K, F$ is an ergodic decomposition of $X \times S \times K$. Indeed, if $T$ is a Borel function from $X \times S \times K$ into itself satisfying $T(x, s, k)=\left(x, a\left(p(x) k^{-1}, k\right)^{-1} s, k\right)$ a.e. $s$ a.e. $(x, k)$, then

$$
\begin{aligned}
T(x \cdot g, s, k \phi(x, g)) & =\left(x \cdot g, a\left(p(x) k^{-1}, k \phi(x, g)\right)^{-1} s, k \phi(x, g)\right) \\
& =\left(x \cdot g,\left(a\left(p(x) k^{-1}, k\right) a(p(x), \phi(x, g))\right)^{-1} s, k \phi(x, g)\right) \\
& =\left(x, a\left(p(x) k^{-1}, k\right)^{-1} s, k\right) \cdot g
\end{aligned}
$$

a.e. $s$ a.e. $(k, x, g)$. Thus $G \circ T^{-1}$ where $G(x, s, k)=\left(p(x) k^{-1}, s\right)$ gives an ergodic decomposition of $W \times K$. But $F=G \circ T^{-1}$. Thus as seen in the appendix $Y_{\tilde{h}}=Y$ $\times S$ and there exist a strict $K$ action on $Y \times S$ and a strict $K$ extension $\sigma_{\tilde{h}}: Y_{\tilde{h}} \rightarrow Y$ satisfying $F\left(x, s, k_{1}^{-1} k\right)=F(x, s, k) \cdot k_{1}$ and $\sigma_{\tilde{h}}(F(x, s, k))=p(x) k^{-1}$ a.e. where 
$Y_{\tilde{h}}$ denotes the Mackey range of $\tilde{h}$. In particular $(y, s) \cdot k_{1}=\left(y \cdot k_{1}, a\left(y, k_{1}\right)^{-1} s\right)$ a.e. $s$ a.e. $\left(y, k_{1}\right)$. Thus $Y_{\tilde{h}}$ is induced by $a$. Q.E.D.

The following theorem is one of a series of theorems relating the spectral properties of the natural representation of an action or extension to the geometric structure of the action. The first significant result obtained along this line is the classical von Neumann-Halmos characterization [4] of transformations with pure point spectrum. To see this fits within the framework of the following theorem we consider a group $G$ acting ergodically on a space $(W, m)$. Let $L$ be the natural unitary representation of $G$ associated with this action and suppose it is a direct sum of one-dimensional representations (pure point spectra). Then there is a unitary transformation $U$ from $L^{2}(W, m)$ into $\oplus H_{i}$, a direct sum of one dimensional Hilbert spaces, with $U L_{g} U^{-1}=\sum \lambda^{i}$ where $\lambda^{i}$ are characters of $G$. Thus $\phi(g)=$ $\left(\lambda_{i}(g)\right)$ is a homomorphism of $G$ whose topological range closure is a compact abelian subgroup $K$ of the unitary group of $\oplus H_{i}$. Define $A(k)=U^{-1} k U$. It follows that $A(k \cdot g)=A(x) L_{g}$. Thus ind ${ }_{W \times G}^{G} 1_{\mid K \times{ }_{\phi} G} \simeq I$. The following theorem shows the $G$ space $W$ is a pull back of an ergodic and thus transitive $K$ space $K / H$. Thus $W$ is a compact abelian group and the $G$ action on $W$ is given by translation composed with a homomorphism $\phi$ having dense range in $W$.

Similarly Zimmer's structure theorem (4.3 of [20]) on extensions with relative spectrum is related to the following theorem and Theorem 2.1 of [3].

Normal actions and normal ergodic extensions were defined in [20] and were characterized in [23 and 2]. They are defined in terms of the natural bundle representation and they too can be set in the context of the following theorem.

THEOREM 2.6. Let $h=(p, \phi)$ be a homomorphism between an ergodic $G$ action on $(X, \mu)$ and an ergodic $K$ action on $(Y, \nu)$. Suppose $h$ has dense range. Let $(W, \lambda)$ be an extension of $(X, \mu)$. Then $W$ is isomorphic over $X$ to an extension $X \times_{h} Y^{\prime}$ where $Y^{\prime}$ is an extension of $Y$ iff $\operatorname{ind}_{W \times G}^{X \times G} 1_{\mid X \times_{\phi} K \times G} \simeq I$.

Proof. First suppose $W \simeq X \times_{h} Y^{\prime}$ for some $K$ space $\left(Y^{\prime}, \nu^{\prime}\right)$ which extends $(Y, \nu)$. Thus $Y^{\prime} \simeq Y \times S$ where the $K$ action on $Y^{\prime}$ is that induced by a strict action $a$ of $Y \times K$ on $S$. Thus $W \simeq X \times S$ with action induced by $h^{*} a$. Hence $\operatorname{ind}_{W \times G}^{X \times G} 1$ is equivalent to the cocycle representation $(x, h) \mapsto L_{h^{*} a(x, g)}$. Therefore, ind $_{W \times G}^{X \times G} 1_{\mid \times_{\phi} K \times G}$ is equivalent to the cocycle representation $(x, k, g) \mapsto L_{h^{*} a(x, g)}$. Therefore ind $W_{W \times G}^{X \times G} 1_{\mid X \times_{\phi} K \times G} \simeq I$ iff the cocycle $(x, k, g) \mapsto h^{*} a(x, g) \in \mathscr{I}(S, m)$ is equivalent to the identity cocycle. To see this let $U(x, k)=a\left(p(x) k^{-1}, k\right)$. Then

$$
\begin{aligned}
U(x \cdot g, k \phi(x, g)) & =a\left(p(x \cdot g) \phi(x, g)^{-1} k^{-1}, k \phi(x, g)\right) \\
& =a\left(p(x) k^{-1}, k \phi(x, g)\right) \\
& =a\left(p(x) k^{-1}, k\right) a(p(x), \phi(x, g))=U(x, k) h^{*} a(x, g) .
\end{aligned}
$$

Conversely, suppose $(W, \lambda)$ is an extension of $(X, \mu)$ and $\operatorname{ind}_{W \times G}^{X \times G} 1_{\mid X \times{ }_{\phi} K \times G}$ is equivalent to the identity. We may assume $W$ is induced by a strict action $b$ of $X \times G$ on $(S, m)$. Then ind $1(x, g)=L_{b(x, g)}$. Thus ind $1_{\mid X \times_{\phi} K \times G}$ is the cocycle representation $(x, k, g) \mapsto L_{b(x, g)}$. Since it is equivalent to the identity cocycle 
representation, there is a strongly Borel unitary operator valued function $U$ on $X \times K$ satisfying

$$
U(x \cdot g, k \phi(x, g))=U(x, k) L_{b(x, g)} \text { a.e. }(x, k, g) .
$$

Let $L^{\infty}(S)$ be the von Neumann algebra of bounded multiplication operators on $L^{2}(S, m)$. Define $\mathscr{V}(x, k)=U(x, k) L^{\infty}(S) U(x, k)^{-1}$. By results from [1], this is a Borel map into the space of von Neumann algebras $\mathscr{V}$ on $L^{2}(S)$, where the Borel structure on the space of $\mathscr{V}$ 's is standard and the action $(\mathscr{V}, U) \rightarrow U^{-1} \mathscr{V} U$ is Borel, $U$ belonging to the unitary group of $L^{2}(S)$. Furthermore,

$$
\begin{aligned}
\mathscr{V}(x \cdot g, k \phi(x, g)) & =U(x, k) L_{b(x, g)} L^{\infty}(S) L_{b(x, g)}^{-1} U(x, k)^{-1} \\
& =U(x, k) L^{\infty}(S) U(x, k)^{-1} \\
& =L^{\infty}(S) \text { a.e. }(x, k, g) .
\end{aligned}
$$

Thus, since $(x, k) \rightarrow p(x) k^{-1}$ is the ergodic decomposition of $X \times_{\phi} K$ as a $G$ space, there is a Borel map $y \rightarrow \overline{\mathscr{V}}(y)$ on $Y$ satisfying

$$
\overline{\mathscr{V}}\left(p(x) k^{-1}\right)=\mathscr{V}(x, k) \text { a.e. }(x, k) .
$$

This implies the set $W=\left\{(y, V): V \overline{\mathscr{V}}(y) V^{-1}=L^{\infty}(S)\right\}$ is Borel and $\{y:(y, V) \in$ $W$ for some unitary operator $V\}$ is conull. By the von Neumann selection theorem, there exists a Borel map $V$ on $Y$ satisfying

$$
V(y) \overline{\mathscr{V}}(y) V(y)^{-1}=L^{\infty}(S) \text { a.e. } y .
$$

Define $U^{\prime}(x, k)=V\left(p(x) k^{-1}\right) U(x, k)$. Then

$$
\begin{aligned}
U^{\prime}(u, k) L^{\infty}(S) U^{\prime}(x, k)^{-1} & =V\left(p(x) k^{-1}\right) \mathscr{V}(x, k) V\left(p(x) k^{-1}\right)^{-1} \\
& =L^{\infty}(S) \text { a.e. }(x, k) .
\end{aligned}
$$

Hence by redefining $U^{\prime}$ on a set of measure 0 and replacing $U$ by $U^{\prime}$ we may assume

$$
U(x, k) L^{\infty}(S) U(x, k)^{-1}=L^{\infty}(S) \text { for all }(x, k)
$$

and

$$
U(x \cdot g, k \phi(x, g))=U(x, k) L_{b(x, g)} \quad \text { a.e. }(x, k, g) .
$$

By (2.4a), it follows there is a Borel function $\Psi: X \times K \rightarrow \mathscr{I}(S, m)$ satisfying

$$
U(x, k) M_{f} U(x, k)^{-1}=M_{f} \circ \Psi(x, k)^{-1}
$$

for each $f$ in $L^{\infty}(S)$. But by (2.4b)

$$
\begin{aligned}
M_{f \circ \Psi(x \cdot g, k \phi(x, g))^{-1}} & =U(x, k) L_{b(x, g)} M_{f} L_{b(x, g)}^{-1} U(x, k)^{-1} \\
& =U(x, k) M_{f \circ b(x, g)^{-1}} U(x, k)^{-1} \\
& =M_{f \circ b(x, g)^{-1} \circ \Psi(x, k)^{-1} .}
\end{aligned}
$$

Thus

$$
\psi(x \cdot g, k \phi(x, g))=\psi(x, k) \circ b(x, g) \quad \text { a.e. }(x, k, g) .
$$


That is, $b_{\mid X \times{ }_{\phi} K \times G}$ is cohomologous to the identity. By Proposition $2.1, b$ is cohomologous to $h^{*} a$ for some strict action $a$ of $Y \times K$ on $(S, m)$. Thus the $G$ space induced by $b$ is isomorphic over $X$ to that induced by $h^{*} a$. In particular, $W$ is isomorphic to the fibered skew product of $X$ with the $K$ space $Y^{\prime}$ induced by $a$. Q.E.D.

Corollary 2.7. Let $\phi: X \times G \rightarrow H$ be a cocycle. Let $(W, \lambda)$ be an extension of $(X, \mu)$. Then $W$ is isomorphic over $X$ to a fibered skew product action of $X$ with an extension of the Mackey range of $\phi$ iff

$$
\operatorname{ind}_{W \times G}^{X \times G} 1_{\mid X \times_{\phi} H \times G} \simeq I .
$$

The following result coalesces many of the notions of this paper and shows only appropriate pull backs of cocycle representations induce representations with trivial restrictions.

THEOREM 2.8. Let $h=(p, \phi)$ be a homomorphism with dense range between the ergodic $G$ space $(X, \mu)$ and the ergodic $K$ space $(Y, \nu)$. Suppose $(W, \lambda)$ is an extension of $X$ and $\pi$ is a cocycle representation of the $G$ space $W$ satisfying

$$
\text { ind }_{W \times G}^{X \times G} \pi_{\mid X \times_{\phi} K \times G} \simeq I \text {. }
$$

Then there exists a strict action a of $Y \times K$ on some space $(S, m)$ such that $W$ is isomorphic over $X$ to the $G$ space induced by $h^{*} a$. Furthermore, if $\mathbf{h}$ is the restriction of $h$ to $W \times G$, the Mackey range $Y_{\mathbf{h}}$ of $\mathbf{h}$ is a $K$ space induced by the action a and the representation $\pi$ is equivalent to a cocycle representation of the form $\left(\mathbf{h}^{\prime}\right)^{*} \pi^{\prime}$ where $\pi^{\prime}$ is a cocycle representation of the $K$ space $Y_{\mathbf{h}}$ and

$$
\begin{array}{ccc}
W \times G & \stackrel{\mathbf{h}}{\rightarrow} & Y \times K \\
\searrow \mathbf{h} & & \nearrow \\
& Y_{\mathbf{h}} \times K &
\end{array}
$$

is the factorization of $\mathbf{h}$ through its Mackey range.

Proof. We may assume $W=X \times S, \lambda=\mu \times m$ and the action of $G$ on $W$ satisfies $(x, s) \cdot g=\left(x \cdot g, b(x, g)^{-1} s\right)$ a.e. $s$ a.e. $(x, g)$, where $b$ is a strict action of $X \times G$ on $(S, m)$. Let $R=\operatorname{ind}_{W \times G}^{X \times G} \pi$. Then $R$ is the cocycle representation on $L^{2}(S, m)$ defined by

$$
R(x, g) f(s)=\frac{d m \circ b(x, g)^{-1}}{d m}(s)^{1 / 2} \pi(x, s, g) f\left(b(x, g)^{-1} s\right) .
$$

Since $R_{\mid X \times{ }_{\phi} K \times G} \simeq I$, there exists a strongly Borel function $U$ on $X \times K$ satisfying

$$
U(x \cdot g, k \phi(x, g))=U(x, k) R(x, g) \text { a.e. }(x, k, g) \text {. }
$$

Now let $L^{\infty}(S)$ be the von Neumann algebra of bounded multiplication operators $M_{f}$ on $L^{2}(S, \mathscr{H}), \mathscr{H}$ the Hilbert space for $\pi$; and set

$$
\mathscr{V}(x, k)=U(x, k) L^{\infty}(S) U(x, k)^{-1}
$$


Since for each $f, R(x, g) M_{f} R(x, g)^{-1}=M_{f \circ b(x, g)^{-1}}$, one sees $\mathscr{V}(x \cdot g, k \phi(x, g))$ $=\mathscr{V}(x, k)$ a.e. $(x, k, g)$. Using the same argument as in the proof of Theorem 2.6, we may assume $U(x, k) L^{\infty}(S) U(x, k)^{-1}=L^{\infty}(S)$ for all $(x, k)$. This implies there is a Borel function $\Psi: X \times K \rightarrow \mathscr{I}(S, m)$ such that each $U(x, k) L_{\psi(x, k)}^{-1}$ centralizes $L^{\infty}(S)$. Thus there is a function $(x, k) \mapsto f_{x, k}$ such that each $f_{x, k}$ is the a.e. $s$ unique Borel function on $S$ with values in the unitary group $\mathscr{U}(\mathscr{H})$ of $\mathscr{H}$ satisfying $U(x, k)=M_{f_{x, k}} L_{\psi(x, k)}$. From (2.5) and (2.6), one obtains

$$
\begin{aligned}
M_{f_{x \cdot g, k \phi(x, g)}} \circ L_{\psi(x \cdot g, k \phi(x, g))} & =M_{f_{x, k}} L_{\psi(x, k)} R(x, g) \\
& =M_{f_{x, k}} M_{\pi\left(x, \psi(x, g)^{-1}(\cdot), g\right)} L_{\psi(x, k) \circ b(x, g)} .
\end{aligned}
$$

Hence we see

$$
\psi(x \cdot g, k \phi(x, g))=\psi(x, k) b(x, g) \quad \text { a.e. }(x, k, g)
$$

and

$$
f_{x \cdot g, k \phi(x, g)}(s)=f_{x, k}(s) \pi\left(x, \psi(x, k)^{-1} s, g\right) \text { a.e. } s \text { a.e. }(x, k) .
$$

Equation (2.7a) shows the action $b$ restricted to $X \times{ }_{\phi} K \times G$ is cohomologous to the identity cocycle. By Lemmas A, B, and C of the proof of Proposition 2.1 and the remarks after Theorem 1.2, we see there exists a strict action $a$ of $Y \times K$ on $(S, m)$ and a Borel map $\Psi^{\prime}$ on $X$ with values in $\mathscr{I}(S, m)$ satisfying

$$
\psi\left(x, k_{1}\right) \psi\left(x, k_{2}^{-1} k_{1}\right)^{-1}=a\left(p(x) k_{1}^{-1}, k_{2}\right) \text { a.e. }\left(x, k_{1}, k_{2}\right)
$$

and

$$
\psi(x, k)=a\left(p(x) k^{-1}, k\right) \psi^{\prime}(x) \text { a.e. }(x, k)
$$

In particular

$f_{x \cdot g, k \phi(x, g)}(s)=f_{x, k}(s) \pi\left(x, \psi^{\prime}(x)^{-1} a\left(p(x) k^{-1}, k\right)^{-1} s, g\right)$ a.e. $s$ a.e. $(x, k, g)$.

Furthermore, the end of the proof of Proposition 2.1 shows $b(x, g)=$ $\psi^{\prime}(x)^{-1} a(p(x), \phi(x, g)) \psi^{\prime}(x \cdot g)$. This shows the map $\Phi(x, s)=\left(x, \psi^{\prime}(x) s\right)$ satisfies

$$
\Phi\left(x \cdot g, b(x, g)^{-1} s\right)=\left(x \cdot g, h^{*} a(x, g)^{-1} \psi^{\prime}(x) s\right) \text { a.e. } s \text { a.e. }(x, g) .
$$

Thus $\Phi$ yields an isomorphism over $X$ between $W$ and the $G$ space induced by $h^{*} a$. Under this isomorphism the representation $\pi$ of the $G$ space $W$ corresponds to the representation $\pi^{\prime}$ of the $G$ space induced by $h^{*} a$ defined by $\pi^{\prime}(x, s, g)=$ $\pi\left(\Phi^{-1}(x, s), g\right)=\pi\left(x, \psi^{\prime}(x)^{-1} s, g\right)$. From (2.9) we obtain

$$
f_{x \cdot g, k \phi(x, g)}(s)=f_{x, k}(s) \pi^{\prime}\left(x, a\left(p(x) k^{-1}, k\right)^{-1} s, g\right) \text { a.e. } s \text { a.e. }(x, k, g) \text {. }
$$

Since $x, k \mapsto M_{f_{x, k}}$ is strongly Borel, there is a Borel function $f$ on $X \times K \times S$ satisfying

$$
\begin{aligned}
& f(x \cdot g, k \phi(x, g), s) \\
& \quad=f(x, k, s) \pi^{\prime}\left(x, a\left(p(x) k^{-1}, k\right)^{-1} s, g\right) \text { a.e. } s \text { a.e. }(x, k, g) .
\end{aligned}
$$


Define $A(x, s, k)=f\left(x, k, a\left(p(x) k^{-1}, k\right) s\right)$. Then $A$ is a Borel function and

$$
\begin{aligned}
A(x & \left.\cdot g, h^{*} a(x, g)^{-1} s, k \phi(x, g)\right) \\
& =f\left(x \cdot g, k \phi(x, g), a\left(p(x \cdot g) \phi(x, g)^{-1} k^{-1}, k \phi(x, g)\right) h^{*} a(x, g)^{-1} s\right) \\
& =f\left(x \cdot g, k \phi(x, g), a\left(p(x) k^{-1}, k \phi(x, g)\right) a(p(x), \phi(x, g))^{-1} s\right) \\
& =f\left(x \cdot g, k \phi(x, g), a\left(p(x) k^{-1}, k\right) s\right) \\
& =f\left(x, k, a\left(p(x) k^{-1}, k\right) s\right) \pi^{\prime}(x, s, g) \\
& =A(x, s, k) \pi^{\prime}(x, s, g) \quad \text { a.e. }(x, s, k, g) .
\end{aligned}
$$

Thus $\pi_{\mid X \times S \times{ }_{\phi} K \times G} \simeq I$. Since $\mathbf{h}=\left(\sigma_{h}, i\right) \circ \mathbf{h}^{\prime}$ and $\mathbf{h}^{\prime}$ has dense range by Proposition A.11, we see by Corollary 2.2, that $\pi^{\prime} \simeq\left(\mathbf{h}^{\prime}\right)^{*} \pi^{\prime \prime}$. That $Y_{\mathbf{h}}$ is the $K$ space induced by $a$ follows by Proposition 2.5. Q.E.D.

Appendix. The intent of this appendix is twofold. The first is to present the results necessary for the development of this paper. The second is to show that one can make strict all actions, equivariant maps, and homomorphisms with which we shall deal simultaneously. Our approach follows Ramsay [16]. The main tool is the following well-known selection theorem.

Lemma A.1 (Federer Morse Selection Theorem). Suppose $X$ is a $\sigma$-compact metric space and $f$ is a continuous function on $X$ with values in a complete separable metric space $Y$. Then there exists a Borel function $\theta: f(X) \rightarrow X$ satisfying $f(\theta(y))=y$.

In order to apply this selection theorem we need the following fact about analytic subsets of complete separable metric spaces. For a proof see Theorem 3.2 of [12].

Lemma A.2. Let $A$ be an analytic subset of a complete separable metric space $X$. Suppose $\mu$ is a $\sigma$-finite measure on $X$. Then there exists a $\sigma$-compact set $B \subseteq A$ satisfying $\mu(A-B)=0$.

We shall also use the following lemma.

LemmA A.3. Suppose $X, Y$, and $Z$ are Borel spaces and $X$ and $Z$ are countably generated. Suppose $\mu$ is a $\sigma$-finite measure on $X$ and $f: X \times Y \rightarrow Z$ is a Borel function. Then $Y_{0}=\{y: f(x, y)$ is $\mu$ a.e. $x$ a constant $\}$ is a Borel subset of $Y$; and if $f_{0}$ is the function defined on $Y_{0}$ by $f_{0}(y)=c$ if $f(x, y)=c \mu$ a.e. $x$, then $f_{0}$ is a Borel function on $Y_{0}$.

Proof. We may assume $\mu$ is a probability measure and $Z$ is a subset of $[0,1]$ with the relative Borel structure. Let $E_{1}, E_{2}, E_{3}, \ldots$ be a countable generating sequence of sets for the Borel structure on $X$. Then $y \in Y_{0}$ iff $\mu\left(E_{j}\right) \int_{E_{i}} f(x, y) d \mu(x)=$ $\mu\left(E_{i}\right) \int_{E_{j}} f(x, y) d \mu(x)$ for all $i, j$. Thus $Y_{0}$ is a Borel set. Furthermore $f_{0}(y)=$ $\int f(x, y) d \mu(x)$ for $y$ in $Y_{0}$. Thus $f_{0}$ is a Borel function.

Lemma A.4. Let $(X, \mu)$ be a strict $G$ space. Suppose $X_{0}$ is a conull Borel subset of $X$. Then $X_{0}$ contains a conull Borel subset $E$ such that

(a) $[E]=E \cdot G$ is a Borel subset of $X$ and

(b) there exists a Borel map $\theta$ from $[E]$ into $G$ such that $\theta(x)=e$ whenever $x$ is in $E$ and $x \cdot \theta(x)^{-1} \in E$ for all $x$ in $[E]$. 
Proof. We may assume $X=U^{G}$, the universal $G$ space. Hence $X$ is a complete separable metric space, and the action is continuous. By Lemma A.2, $X_{0}$ contains a conull $\sigma$-compact set $E$. Thus $[E]=E \cdot G$ is $\sigma$-compact and therefore Borel. The map $f: E \times G \rightarrow E \cdot G$ defined by $f(x, g)=x \cdot g$ is continuous on the $\sigma$-compact set $E \times G$. By Federer Morse, there exists a Borel function $\psi: E \cdot G \rightarrow E \times G$ satisfying $f(\psi(x))=x$. Let $\psi=(q, \theta)$. Then $q(x) \cdot \theta(x)=x$ on $[E]$. Thus $x$. $\theta(x)^{-1}=q(x) \in E$ for all $x$. Redefine $\theta$ on $E$ by $\theta(x)=e$. Q.E.D.

Proposition A.5. Let $(X, \mu)$ be a strict $G$ space. Suppose $a$ is an action of $X \times G$ on the space $(S, m)$. Furthermore, suppose $x \mapsto E(x)$ is a Borel map on $X$ with values in the measure algebra $M(S, m)$ defined by $m$ satisfying $E(x \cdot g)=a(x, g)^{-1} E(x)$ a.e. $(x, g)$. Then there is a Borel function $F: X \rightarrow M(S, m)$ satisfying $F(x)=E(x)$ a.e. $x$ and $F(x \cdot g)=a(x, g)^{-1} F(x)$ for all $(x, g)$.

Proof. Let $W=\left\{(x, g): E(x \cdot g)=a(x, g)^{-1} E(x)\right\}$. Then $W$ is a Borel set which is conull in $X \times G$. Furthermore, if $\left(x, g_{1}\right)$ and $\left(x g_{1}, g_{2}\right)$ are in $W$, then $\left(x, g_{1} g_{2}\right)$ belongs to $W$. By Lemma 5.2 of [13], there is a conull Borel set $E_{0}$ in $X$ such that if $x$ and $x \cdot g$ belong to $E_{0}$, then $(x, g)$ is in $W$. By Lemma A.4, we may assume $\left[E_{0}\right]=E_{0} \cdot G$ is Borel and there exists a Borel map $\theta:\left[E_{0}\right] \rightarrow G$ satisfying $x \theta(x)^{-1} \in E_{0}$ and $\theta(x)=e$ for $x$ in $E_{0}$.

Define $F(x)=a\left(x, \theta(x)^{-1}\right) E\left(x \cdot \theta(x)^{-1}\right)$ if $x \in\left[E_{0}\right]$ and $F(x)=\phi$ otherwise. Clearly $F(x)=E(x)$ if $x \in E_{0}$ and $F(x \cdot g)=a(x, g)^{-1} F(x)$ for $x \notin\left[E_{0}\right]$. Suppose $x \in\left[E_{0}\right]$. Then

$$
\begin{aligned}
F(x \cdot g) & =a\left(x \cdot g, \theta(x \cdot g)^{-1}\right) E\left(x \cdot g \theta(x \cdot g)^{-1}\right) \\
& =a\left(x \cdot g, \theta(x \cdot g)^{-1}\right) E\left(x \cdot \theta(x)^{-1} \theta(x) g \theta(x \cdot g)^{-1}\right) \\
& =a\left(x \cdot g, \theta(x \cdot g)^{-1}\right) a\left(x \cdot \theta(x)^{-1}, \theta(x) g \theta(x \cdot g)^{-1}\right)^{-1} E\left(x \cdot \theta(x)^{-1}\right) \\
& =a\left(x \cdot g, g^{-1} \theta(x)^{-1}\right) E\left(x \cdot \theta(x)^{-1}\right) \\
& =a\left(x \cdot g, g^{-1}\right) a\left(x, \theta(x)^{-1}\right) E\left(x \cdot \theta(x)^{-1}\right) \\
& =a(x, g)^{-1} F(x)
\end{aligned}
$$

since $x \cdot \theta(x)^{-1} \in E_{0}$ and $x \cdot g \theta(x \cdot g)^{-1} \in E_{0}$. Q.E.D.

The following propositions can be proved using an argument essentially the same as that used in Proposition A.5. Moreover they are essentially equivalent to Theorems 3.4 and 3.2 in [16].

Proposition A.6. Let $(X, \mu)$ and $(Y, \nu)$ be strict $G$ spaces. Suppose $y \mapsto \lambda_{y}$ is $a$ Borel map from $Y$ into the space of $\sigma$-finite measures on $X$ with the weak Borel structure. Furthermore suppose $\lambda_{y} \cdot g \sim \lambda_{y \cdot g}$ a.e. $(y, g)$ where $(\lambda \cdot g)(E)$ is defined to be $\lambda\left(E \cdot g^{-1}\right)$. Then there exists a Borel function $y \mapsto \lambda_{y}^{\prime}$ such that $\lambda_{y}^{\prime}=\lambda_{y}$ a.e. $y$ and $\lambda_{y \cdot g}^{\prime} \sim \lambda_{y}^{\prime} \cdot g$ for all $(y, g)$.

Proposition A.7. Let $(X, \mu)$ be a strict ergodic $G$ space. Let $(Y, \nu)$ be a strict $K$ space. Suppose $h$ is a Borel mapping from $X \times G$ into $Y \times K$ satisfying

$$
h\left(x, g_{1} g_{2}\right)=h(x, g) h\left(x g_{1}, g_{2}\right) \text { a.e. }\left(x, g_{1}, g_{2}\right) \text {. }
$$


Then there exists a Borel mapping $h_{0}$ from $X \times G$ into $Y \times K$ equal to $h$ a.e. and satisfying (A.1) for all $\left(x, g_{1}, g_{2}\right)$.

Corollary A.8. Suppose $(X, \mu)$ is a $G$ space and $\phi$ is a Borel mapping on $X \times G$ with values in a Polish group $K$ satisfying $\phi\left(x, g_{1} g_{2}\right)=\phi\left(x, g_{1}\right) \phi\left(x g_{1}, g_{2}\right)$ a.e. $\left(x, g_{1}, g_{2}\right)$. Then there is a cocycle $\phi_{0}$ equal to $\phi$ a.e. $(x, g)$.

The Mackey Range. Suppose $(X, \mu)$ is a strict ergodic $G$ space and $(Y, \nu)$ is a strict ergodic $K$ space. Let $h=(p, \phi)$ be a homomorphism from $X \times G$ into $Y \times K$. Our intent here is to develop the notion of the Mackey range of $h$. We show it will only be necessary to redefine $h$ on a set of measure 0 in order to make all the relative mappings and actions strict.

The first step is to define a strict action of $G$ on $X \times K$ by $(x, k) \cdot g=(x$. $g, k \phi(x, g))$. Then there exists an ergodic decomposition $F: X \times K \rightarrow Y_{h}$ of the $G$ space $X \times K$. This mapping is essentially $G$ invariant. Furthermore, for each $k_{1}$ in $K$, the map $(x, k) \mapsto\left(x, k_{1} k\right)$ is a strict $G$ space isomorphism of $X \times K$. Thus the mapping $(x, k) \mapsto F\left(x, k_{1} k\right)$ gives another ergodic decomposition of the $G$ space $X \times K$. By the uniqueness of the ergodic decomposition, there exists a Borel map $\psi\left(k_{1}\right) \in \mathscr{I}\left(Y_{h}, \nu_{h}\right)$, where $\nu_{h}=F_{*}\left(\lambda \times m_{K}\right)$, satisfying $\psi\left(k_{1}\right) F(x, k)=F\left(x, k_{1} k\right)$ a.e. $(x, k)$. It follows that $\psi$ is a Borel function and $\psi\left(k_{1} k_{2}\right)=\psi\left(k_{1}\right) \psi\left(k_{2}\right)$. Hence $\psi$ defines an action of $K$ on $\left(Y_{h}, \nu_{h}\right)$. By [6] there is a strict $K$ action on $\left(Y_{h}, \nu_{h}\right)$ with fixed points satisfying $y \cdot k=\psi(k)^{-1} y$ a.e. $y$ for each $k$. Thus $F(x, k) \cdot k_{1}=$ $F\left(x, k_{1}^{-1} k\right)$ a.e. $(x, k)$ for each $k_{1}$.

Let $y_{0}$ be a fixed point for the action of $K$ on $Y_{h}$. Then by Lemma A.3 $W_{1}=\{(x, k): g \mapsto F(x \cdot g, k \phi(x, g))$ is a constant a.e. $g\}$ is a conull Borel set and the function $F^{\prime}$ defined on $W_{1}$ by $F^{\prime}(x, k)=F(x \cdot g, k \phi(x, g))$ a.e. $g$ is Borel. Define $F^{\prime}$ on $X \times K-W_{1}$ by $F^{\prime}(x, k)=y_{0}$. Then $F^{\prime}(x, k)=F(x, k)$ a.e. Suppose $(x, k)$ belongs to $W_{1}$. Then if $g \in G$,

$$
F\left(x \cdot g_{1}, k \phi\left(x, g_{1}\right)\right)=F\left(x \cdot g g_{1}, k \phi\left(x, g g_{1}\right)\right) \text { a.e. } g_{1} \text {. }
$$

Thus

$$
F\left(x \cdot g_{1}, k \phi\left(x, g_{1}\right)\right)=F\left(x \cdot g \cdot g_{1}, k \phi(x, g) \cdot \phi\left(x g, g_{1}\right)\right) \text { a.e. } g_{1} \text {. }
$$

Thus $(x \cdot g, k \phi(x, g)) \in W_{1}$ and $F^{\prime}(x \cdot g, k \phi(x, g))=F^{\prime}(x, k)$. Thus $F^{\prime}$ is $G$ invariant. Replacing $F$ by $F^{\prime}$ we see one may assume $F$ is strictly $G$ invariant.

Let $W_{2}=\left\{\left(x, k_{1}\right): F\left(x, k^{-1} k_{1}\right) \cdot k^{-1}\right.$ is constant a.e. $\left.k\right\}$. Then Lemma A.3 shows $W_{2}$ is a conull Borel subset and the function $F^{\prime}$ defined on $W_{2}$ by $F^{\prime}\left(x, k_{1}\right)=$ $F\left(x, k^{-1} k_{1}\right) \cdot k$ a.e. $k$ is Borel. Define $F^{\prime}$ on $X \times W-W_{2}$ by $F^{\prime}(x, k)=y_{0}$. Then $F^{\prime}$ is Borel, strictly $G$ invariant, $F^{\prime}\left(x, k^{-1} k_{1}\right)=F^{\prime}\left(x, k_{1}\right) \cdot k$ for all $x, k, k_{1}$, and $F^{\prime}\left(x, k_{1}\right)=F\left(x, k_{1}\right)$ a.e. $\left(x, k_{1}\right)$. Replacing $F$ by $F^{\prime}$, we thus have $F$ is a strictly $G$ invariant Borel map from $X \times K$ into $\left(Y_{h}, \nu_{h}\right)$ which gives an ergodic decomposition $X \times K$ and satisfies $F\left(x, k_{1}^{-1} k\right)=F(x, k) \cdot k_{1}$ for all $x, k_{1}$, and $k_{2}$.

Next, consider the mapping $f$ from $X \times K$ into $Y$ defined by $f(x, k)=p(x) k^{-1}$. Since $h$ is a homomorphism, $f$ is strictly $G$ invariant. Thus, since $F$ is an ergodic decomposition of $X \times K$, there exists a Borel map $\sigma_{h}$ from $Y_{h}$ into $Y$ satisfying $\sigma_{h}(F(x, k))=p(x) k^{-1}=f(x, k)$ a.e. $(x, k)$. This implies $\sigma_{h}(y \cdot k)=\sigma_{h}(y) \cdot k$ a.e. $y$ for each $k$. 
Let $Y_{0}=\left\{y \in Y_{h}: \sigma_{h}(y \cdot k) k^{-1}\right.$ is constant a.e. $\left.k\right\}$. By Lemma A.3, $Y_{0}$ is a Borel set and the mapping $\sigma_{h}^{\prime}$ defined on $Y_{0}$ by $\sigma_{h}^{\prime}(y)=\sigma_{h}(y \cdot k) k^{-1}$ a.e. $k$ is Borel. Clearly $Y_{0}$ is $K$ invariant and $\sigma_{h}^{\prime}=\sigma_{h}$ a.e. on $Y_{0}$. Furthermore, if $y \in Y_{0}$, then for a.e. $k$,

$$
\sigma_{h}^{\prime}\left(y \cdot k_{1}\right)=\sigma_{h}\left(y \cdot k_{1} k\right) k^{-1} k_{1}^{-1} k_{1}=\sigma_{h}^{\prime}(y) k_{1} .
$$

Thus $\sigma_{h}^{\prime}$ is strictly $K$ equivariant on the $K$ invariant conull Borel subset $Y_{0}$ of $Y_{h}$. Redefine $\sigma_{h}$ to be $\sigma_{h}^{\prime}$ on $Y_{0}$.

Finally let $W_{3}=\left\{(x, k): F(x, k) \in Y_{0}\right.$ and $\left.\sigma_{h}(F(x, k))=p(x) k^{-1}\right\} . W_{3}$ is a conull $G \times K$ invariant Borel subset of $X \times K$. Since $W_{3}$ is $K$ invariant, $W_{3}=X_{0}$ $\times K$ for some Borel subset $X_{0}$ of $X$. Since $W_{3}$ is $G$ invariant, $X_{0}$ is a $G$ invariant conull Borel subset of $X$. Replace $Y_{h}$ by $Y_{0}$.

We thus may conclude the following. Suppose $h$ is a homomorphism from $X \times G$ into $Y \times K$. Then there exist a conull $G$ invariant Borel subset $X_{0}$ of $X$, a strict $K$ space $\left(Y_{h}, \nu_{h}\right)$, a Borel $G$ invariant map $F$ from $X_{0} \times K$ into $Y_{h}$, and a $K$ equivariant Borel map $\sigma_{h}$ from $Y_{h}$ into $Y$ satisfying

(a) $F$ is an ergodic decomposition of $X_{0} \times K$,

(b) $F\left(x, k_{1}^{-1} k\right)=F(x, k) k_{1}$ for all $\left(x, k, k_{1}\right)$, and

(c) $\sigma_{h}(F(x, k))=p(x) k^{-1}$ for all $(x, k)$.

Define $p_{h}(x)=F(x, e)$ for $x$ in $X_{0}$. Then $F(x, k)=p_{h}(x) k^{-1}$ for all $(x, k) . p_{h}$ satisfies $p_{h}(x \cdot g)=p_{h}(x) \cdot \phi(x, g)$ for all $x$ and $g$, since $F$ is $G$ invariant. Thus $h^{\prime}=\left(p_{h}, \phi\right)$ is a multiplicative map from $X_{0} \times G$ into $Y_{0} \times K$.

Proposition A.9. The map $\sigma_{h}:\left(Y_{h}, \nu_{h}\right) \rightarrow(Y, \nu)$ is an ergodic extension.

Proof. First we show the map $\sigma_{h} \times i$ which sends $(y, k)$ to $\left(\sigma_{h}(y), k\right)$ is a homomorphism. Clearly it is multiplicative. Next, note that $Y_{h}$ is an ergodic $K$ space. Indeed, if $E$ is a $K$ invariant Borel subset of $Y_{h}, F^{-1}(E)$ is a $G \times K$ invariant subset of $X_{0} \times K$. It thus has form $E_{0} \times K$ where $E_{0}$ is a $G$ invariant Borel set. Thus $E_{0}$ is null or conull. Thus $F^{-1}(E)$ is null or conull; thus $E$ is $\nu_{h}$ null or conull. Lastly we need to show that if $A$ is an invariant analytic subset of $Y$, $\sigma_{h}^{-1}(A)$ is $\nu_{h}$ null iff $A$ is $\nu$ null. But since $h$ is a homomorphism, $A$ is null iff $p^{-1}(A)$ is null in $X$ iff $\left\{(x, k): p(x) k^{-1} \in A\right\}$ is null in $X_{0} \times K$ iff $\{(x, k)$ : $\left.\sigma_{h}(F(x, k)) \in A\right\}$ is null in $X_{0} \times K$ iff $F^{-1}\left(\sigma_{h}^{-1}(A)\right)$ is null in $X_{0} \times K$ iff $\sigma_{h}^{-1}(A)$ is null in $Y_{h}$.

To show $\sigma_{h}$ is an extension, we must show $\sigma_{h_{*}} \nu_{h} \sim \nu$. Let $\lambda=\sigma_{h_{*}} \nu_{h}=\nu_{h} \circ \sigma_{h}^{-1}$. Then $\lambda=\lambda_{a}+\lambda_{s}$ where $\lambda_{a} \ll \nu$ and $\lambda_{s} \perp \nu$. But $\lambda \cdot k \sim \lambda$. Thus $\lambda \cdot k=\lambda_{a} \cdot k+$ $\lambda_{s} \cdot k$ and $\lambda_{a} \cdot k \ll \nu \cdot k \sim \nu, \lambda_{s} \cdot k \perp \nu \cdot k \sim \nu$. Hence $\lambda_{a} \cdot k \ll \nu$ and $\lambda_{s} \cdot k \perp \nu$. Therefore, $\lambda_{a} \cdot k \sim \lambda_{a}$ and $\lambda_{s} \cdot k \sim \lambda_{a}$. But there exists a Borel set $E$ with $\lambda_{a}=\lambda_{\mid E}$ and $\lambda_{s}=\lambda_{\mid F}$, where $F=Y-E$. Since $\lambda_{a} \cdot k \sim \lambda_{a}$ and $\lambda_{s} \cdot k \sim \lambda_{s}$, one has for each $k, E \cdot k=E$ and $F \cdot k=F \lambda$ essentially. We may thus assume $E$ and $F$ are $K$ invariant. Thus $E$ or $F$ is $\lambda$-null. If $E$ is $\lambda$-null, then $\nu_{h}\left(\sigma_{h}^{-1}(E)\right)=0$ which occurs iff $\nu(E)=0$ since $E$ is invariant and $\sigma_{h} \times i$ is a homomorphism. But then $F$ is conull. This implies $\lambda=\lambda_{s} \perp \nu$. Hence $\nu(F)=0$. But then $\nu(Y)=\nu(E)+\nu(F)$ $=0$. This is a contradiction. Thus $\lambda(F)=0$. Hence $\nu_{h}\left(\sigma_{h}^{-1}(F)\right)=0$. Since $\sigma_{h} \times i$ is 
a homomorphism, $\nu(F)=0$. Therefore $E$ is conull and $\lambda=\lambda_{a} \ll \nu$. But then $\lambda=\int f d \nu$ and $y \cdot k \sim \lambda$ implies $f(y \cdot k)>0$ iff $f(y)>0$. Hence $f(y)>0 \nu$ a.e. $y$. Therefore $\lambda \sim \nu$. Q.E.D.

The major conclusion of the above construction is the following.

THEOREM A.10. Let $h=(p, \phi)$ be a homomorphism between the ergodic $G$ space $(X, \mu)$ and the ergodic $K$ space $(Y, \nu)$. Then there exist a conull $G$ invariant Borel subset $X_{0}$ of $X$, an ergodic extension $\sigma_{h}:\left(Y_{h}, v_{h}\right) \rightarrow(Y, \nu)$, and a Borel mapping $p_{h}$ : $X_{0} \rightarrow Y_{h}$ such that

(1) $\sigma_{h} \circ p_{h}(x)=p(x)$ for all $x$ in $X_{0}$,

(2) $p_{h}(x \cdot g)=p_{h}(x) \phi(x, g)$ for all $(x, g)$ in $X_{0} \times G$,

(3) an ergodic decomposition for $X \times_{\phi} K$ is given by the map $(x, k) \rightarrow p_{h}(x) k^{-1}$, and

(4) $h^{\prime}=\left(p_{h}, \phi\right)$ is a homomorphism of $X_{0} \times G$ into $Y_{h} \times K$.

Proof. All has been established except property (1.8) for the mapping $h^{\prime}$. Let $E$ be an invariant analytic subset of $Y_{h}$. It is null in $Y_{h}$ iff $\left\{(x, k): p_{h}(x) k^{-1} \in E\right\}$ is null in $X \times K$ iff $p_{h}^{-1}(E)$ is null in $X$. Q.E.D.

The $K$ space $\left(Y_{h}, \nu_{h}\right)$ is called the Mackey range of $h$. The homomorphism $\sigma_{h} \times i$ : $Y_{h} \times K \rightarrow Y \times K$ is called the inclusion of the Mackey range in $Y \times K$. The decomposition $h_{\mid X_{0} \times G}=\left(\sigma_{h} \times i\right) \circ h^{\prime}$ is called the factorization of $h$ through the Mackey range of $h$. The homomorphism $h$ is said to have dense range if the extension $\sigma_{h}: Y_{h} \rightarrow Y$ is a $K$ space isomorphism.

REMARK. The homomorphism $h$ has dense range iff the map $(x, k) \rightarrow p(x) k^{-1}$ gives the ergodic decomposition of the $K$ space $X \times_{\phi} K$.

Proposition A.11. The homomorphism $h^{\prime}=\left(p_{h}, \phi\right)$ has dense range in $Y_{h} \times K$.

Proof. The ergodic decomposition for $X \times_{\phi} K$ is the map $(x, k) \mapsto p_{h}(x) k^{-1}$.

\section{REFERENCES}

1. E. G. Effros, The Borel space of von Neumann algebras on a separable Hilbert space, Pacific J. Math. 15 (1963), 1153-1164.

2. R. C. Fabec, Normal ergodic actions and extensions, Israel Math. J. 40 (1981), 175-186.

3. Cocycles, extensions of group actions, and bundle representations, J. Funct. Anal. 56 (1984), 79-98.

4. P. R. Halmos and J. von Neumann, Operator algebras in classical mechanics. II, Ann. of Math. 43 (1942), 332-350.

5. G. W. Mackey, Borel structures in groups and their duals, Trans. Amer. Math. Soc. 85 (1957), $265-311$.

6. __ Point realizations of transformation groups, Illinois J. Math. 6 (1962), 327-335.

7. $\longrightarrow$ Ergodic theory, group theory, and differential geometry, Proc. Nat. Acad. Sci. U.S.A. 50 (1963), 1184-1191.

8. __ Ergodic transformations with a pure point spectrum, Illinois J. Math. 8 (1964), 593-600.

9. __ Ergodic theory and virtual groups, Math. Ann. 166 (1966), 187-207.

10. C. C. Moore, Extensions and cohomology for locally compact groups. III, Trans. Amer. Math. Soc. 221 (1976), 1-33.

11. C. C. Moore and R. J. Zimmer, Groups admitting ergodic actions with generalized discrete spectrum, Invent. Math. 51 (1979), 171-188.

12. K. R. Parthasarathy, Probability measures on metric spaces, Academic Press, New York, 1967.

13. A. Ramsay, Virtual groups and group actions, Adv. in Math. 6 (1971), 253-322. 
14. __ Boolean duals of virtual groups, J. Funct. Anal. 15 (1974), 56-101.

15. ___ Subobjects of virtual groups, Pacific J. Math. 87 (1980), 389-454.

16. __ Topologies on measured groupoids, J. Funct. Anal. 47 (1982), 314-343.

17. C. Series, The Poincare flow of a foliation, Amer. J. Math. 102 (1980), 93-128.

18. J. J. Westman, Virtual group homomorphisms with dense range, Illinois J. Math. 20 (1976), 41-47.

19. R. J. Zimmer, Compact nilmanifold extensions of ergodic actions, Trans. Amer. Math. Soc. 223 (1976), 397-406.

20. __ Extensions of ergodic group actions, Illinois J. Math. 20 (1976), 373-409.

21. __ Ergodic actions with generalized discrete spectrum, Illinois J. Math. 20 (1976), 555-588.

22. __ Cocycles and the structure of ergodic group actions, Israel Math. J. 26 (1977), 214-220.

23. __ Normal ergodic actions, J. Funct. Anal. 25 (1977), 286-305.

24. Orbit spaces of unitary representations, ergodic theory, and simple Lie groups, Ann. of Math. (2) 106 (1977), 573-588.

25. Amenable ergodic group actions and an application of Poisson boundaries of random walks, J. Funct. Anal. 27 (1978), 350-372.

26. 407-428.

Department of Mathematics, Louisiana State University, Baton Rouge, Louisiana 70803 